\title{
Transitional events in the spectrophotometric regime between stripped envelope and superluminous supernovae
}

\author{
S. J. Prentice ${ }^{\circledR},{ }^{1 \star}$ C. Inserra ${ }^{\oplus},{ }^{2}$ S. Schulze ${ }^{\circledR},{ }^{3}$ M. Nicholl, ${ }^{4}$ P. A. Mazzali, ${ }^{5,6}$ S. D. Vergani, ${ }^{7}$ \\ L. Galbany ${ }^{\oplus},{ }^{8,9}$ J. P. Anderson, ${ }^{10}$ C. Ashall, ${ }^{11}$ T. W. Chen, ${ }^{3}$ M. Deckers, ${ }^{1}$ M. Delgado Mancheño, ${ }^{8}$ \\ R. González Díaz, ${ }^{9,12}$ S. González-Gaitán ${ }^{\oplus},{ }^{13}$ M. Gromadzki ${ }^{\oplus},{ }^{14}$ C. P. Gutiérrez,,${ }^{15,16}$ L. Harvey, ${ }^{1}$ \\ A. Kozyreva ${ }^{\odot},{ }^{6}$ M. R. Magee,,${ }^{1}$ K. Maguire ${ }^{\oplus},{ }^{1}$ T. E. Müller-Bravo ${ }^{\oplus},{ }^{17}$ S. Muñoz Torres, ${ }^{8}$ P. J. Pessi, ${ }^{10,18}$ \\ J. Sollerman, ${ }^{3}$ J. Teffs ${ }^{\oplus},{ }^{5}$ J. H. Terwel ${ }^{1}$ and D. R. Young ${ }^{19}$ \\ ${ }^{1}$ School of Physics, Trinity College Dublin, The University of Dublin, Dublin 2, Ireland \\ ${ }^{2}$ School of Physics and Astronomy, Cardiff University, Queens Buildings, The Parade, Cardiff CF24 3AA, UK \\ ${ }^{3}$ The Oskar Klein Centre, Department of Astronomy, Stockholm University, AlbaNova, SE-106 91 Stockholm, Sweden \\ ${ }^{4}$ Birmingham Institute for Gravitational Wave Astronomy and School of Physics and Astronomy, University of Birmingham, Birmingham B15 2TT, UK \\ ${ }^{5}$ Astrophysics Research Institute, Liverpool John Moores University, IC2, Liverpool Science Park, 146 Brownlow Hill, Liverpool L3 5RF, UK \\ ${ }^{6}$ Max-Planck-Institut für Astrophysik, Karl-Schwarzschild-Str 1, D-85748 Garching, Germany \\ ${ }^{7}$ GEPI, Observatoire de Paris, PSL University, CNRS, 5 Place Jules Janssen, F-92190 Meudon, France \\ ${ }^{8}$ Departamento de Física Teórica y del Cosmos, Universidad de Granada, E-18071 Granada, Spain \\ ${ }^{9}$ Institute of Space Sciences (ICE, CSIC), Campus UAB, Carrer de Can Magrans, s/n, E-08193 Barcelona, Spain \\ ${ }^{10}$ European Southern Observatory, Alonso de Córdova 3107, Casilla 19, Santiago, Chile \\ ${ }^{11}$ Institute for Astronomy, University of Hawai'i at Manoa, 2680 Woodlawn Dr., Hawai'i, HI 96822, USA \\ ${ }^{12}$ Instituto Nacional de Astrofísica, Óptica y Electrónica (INAOE), 72840 Tonantzintla, Puebla, Mexico \\ ${ }^{13}$ CENTRA-Centro de Astrofísica e Gravitação and Departamento de Física, Instituto Superior Técnico, Universidade de Lisboa, Avenida Rovisco Pais, \\ P-1049-001 Lisboa, Portugal \\ ${ }^{14}$ Astronomical Observatory, University of Warsaw, Al. Ujazdowskie 4, PL-00-478 Warszawa, Poland \\ ${ }^{15}$ Finnish Centre for Astronomy with ESO (FINCA), FI-20014 University of Turku, Finland \\ ${ }^{16}$ Tuorla Observatory, Department of Physics and Astronomy, FI-20014 University of Turku, Finland \\ ${ }^{17}$ School of Physics and Astronomy, University of Southampton, Southampton, Hampshire SO17 1BJ, UK \\ ${ }^{18}$ Facultad de Ciencias Astronómicas y Geofísicas (FCAG), Universidad Nacional de La Plata (UNLP), Paseo del bosque S/N, 1900, Argentina \\ ${ }^{19}$ Astrophysics Research Centre, School of Mathematics and Physics, Queen's University Belfast, Belfast BT7 1NN, UK
}

Accepted 2021 September 29. Received 2021 September 28; in original form 2021 June 11

\begin{abstract}
ABSTRA CT
The division between stripped-envelope supernovae (SE-SNe) and superluminous supernovae (SLSNe) is not well-defined in either photometric or spectroscopic space. While a sharp luminosity threshold has been suggested, there remains an increasing number of transitional objects that reach this threshold without the spectroscopic signatures common to SLSNe. In this work, we present data and analysis on four SNe transitional between SE-SNe and SLSNe; the He-poor SNe 2019dwa and 2019cri, and the He-rich SNe 2019hge and 2019unb. Each object displays long-lived and variable photometric evolution with luminosities around the SLSN threshold of $M_{r}<-19.8$ mag. Spectroscopically however, these objects are similar to SE-SNe, with line velocities lower than either SE-SNe and SLSNe, and thus represent an interesting case of rare transitional events.
\end{abstract}

Key words: supernovae: general.

\section{INTRODUCTION}

The classification scheme of stripped-envelope supernovae (SE-SNe) is a spectroscopic one that mostly evolved over the latter half of the 20th Century (e.g. Filippenko 1997). Type I and Type II separate Hpoor and $\mathrm{H}$-rich transients. Type Ia separates thermonuclear events from the Type Ibc SE-SNe. The latter group separated into the Herich Type Ib and the He-poor Type Ic. Some SE-SNe, of Type Ic in particular, display broad and blended absorption features in their

^E-mail: sipren.astro@gmail.com spectra indicative of a high specific kinetic energy. These are then further separated into Type Ic-BL. To complicate matters, SE-SNe also include stars that explode with residual $\mathrm{H}$ in their outer layers, these are Type IIb. Prentice \& Mazzali (2017) went further, separating the Type Ic into subclasses which expanded on the absorption width theme by counting the number of certain features in the spectra. This led to a sequence Ic-7 to Ic-3 with increasing specific kinetic energy, thus linking the taxonomic scheme with physical parameters.

Since the discovery of hydrogen-poor superluminous supernovae (SLSNe) in the last decade or so (Quimby et al. 2011), an active topic of research has been establishing how these objects are connected to normal SE-SNe, including $\mathrm{SNe} \mathrm{Ib}$, Ic, IIb, broad line $\mathrm{SNe}$, and 
supernova associated with gamma-ray bursts (For studies of SE-SNe, see Lyman et al. 2016; Prentice et al. 2016, 2019b). 'SLSN' however, is a classification mainly based upon a photometric property (luminosity). Initially, SLSNe were separated in luminosity space from SESNe by an empirical cut: SLSNe are found at $M_{r}=-20$ to $-22 \mathrm{mag}$, making them amongst the most luminous transients in the Universe (Gal-Yam 2012). SE-SNe are found at lower luminosities compared to SLSNe, $M_{r}=-16$ to -19 mag (e.g. Drout et al. 2011; Taddia et al. 2018). With larger and more homogeneous samples, this initially useful magnitude boundary has become blurry: Angus et al. (2019) compared the luminosity distributions of SLSNe from the Dark Energy Survey (DES) with the literature sample of SLSNe, and found that the DES SLSN luminosity distribution peaks at $M_{4000} \sim-19.5 \mathrm{mag}$, while the literature sample peaks at $M_{4000} \sim-20.75 \mathrm{mag}\left(M_{4000}\right.$ is an artificial bandpass centred on $4000 \AA$, see Inserra \& Smartt 2014). De Cia et al. (2018) showed that if one assumes that SE-SNe and SLSNe are transients of similar origin then the luminosity distribution of these objects is smooth and decreases for increasing luminosity.

To have a phenomenological definition of SLSNe similar to those of other SN types, the spectra evolution up to $30 \mathrm{~d}$ post-maximum needs to be probed. A SLSN I spectrum at $30 \mathrm{~d}$ resembles that of a type Ic at peak (e.g. Pastorello et al. 2010; Inserra et al. 2013), exhibiting a photospheric velocity that does not evolve after $30+\mathrm{d}$ in contrast with typical SE-SNe (e.g. Nicholl et al. 2015; Liu, Modjaz \& Bianco 2017a; Inserra et al. 2018b).

Another issue is that SLSNe are rare (Quimby et al. 2013; Frohmaier et al. 2021), and being luminous, they are observed at higher redshift than other SN types making them relatively dim in the observer frame. Consequently, spectroscopic observations with good $\mathrm{S} / \mathrm{N}$ are hard to come by, and long-term monitoring less likely. Thus, the spectroscopic properties of the existing SLSN sample are not well-sampled, with the exception of a few objects (e.g. SNe 2015bn and 2017egm; Nicholl et al. 2016a; Bose et al. 2018).

Inserra et al. (2018b) provided a statistical analysis of SLSNe-I identifying two subclasses based on their photometric and spectroscopic evolution together with the ejecta velocity. Quimby et al. (2018) analysed a large sample of Palomar Transient Factory (PTF) SLSN-I and reported a similar finding with most objects following a similar spectroscopic evolution to PTF12dam or SN 2011ke. They also note that a few objects were 'SLSN-like' but did not fit within this system or had limited data. One of these objects is PTF10ghi (henceforth SN 2010md) which showed $\mathrm{H} / \mathrm{He}$ in its spectra, making it more SN IIb-like (Inserra et al. 2013; Quimby et al. 2018). Indeed, despite the obvious lack of $\mathrm{He}$ in the spectra of most objects, Mazzali et al. (2016) demonstrated through spectral models that He may be present in the post-peak spectra in some SLSNI. They identify iPTF13ajg as a likely candidate and suggested reclassifying this as an SN Ib. Although the photospheric-phase properties of SLSNe are clearly quite heterogeneous, a clustering analysis of their nebular phase spectra by Nicholl et al. (2019) could not identify multiple populations, suggesting their interiors may be similar despite differences in their envelopes and environments.

Another possible distinction between SLSNe (and some energetic SN Ic) and SE-SNe is the observation that they are hosted in different type of galaxies. SLSNe occur in galaxies of lower metallicity and higher specific star formation rate than is typical for SE-SNe (e.g. Wiseman et al. 2020; Schulze et al. 2020a). One possibility is that the SLSN rate is suppressed in galaxies above a metallicity threshold of around one-half solar (Perley et al. 2016; Chen et al. 2017; Schulze et al. 2020a). The high specific star formation rate has been used to point to SLSNe having progenitors with larger $M_{\text {ZAMS }}$ (Leloudas et al. 2015).
It is then clear that the division between SLSNe and normal $\mathrm{SE}-\mathrm{SNe}$ is not as simple as originally proposed. In recent years, wide-field surveys have revealed a few unusual transients including transitional objects, in terms of luminosity or spectra evolution (or both) between SLSNe and normal CC-SNe (Modjaz, Gutiérrez \& Arcavi 2019) such as SN 2017ens (Chen et al. 2018), a transition between an SLSN and an SN IIn or SN 2019hcc (Parrag et al. 2021), a SN II with a classification spectrum typical of a SLSN. In this work, we present data and analysis on four photometrically unusual transients that sit in the 'spectro-luminosity' region between SLSNe and SE-SNe, but which are spectroscopically similar to normal SESNe rather than to most SLSNe. Two of these objects, SNe 2019hge and 2019unb, are spectroscopically similar to both normal He-rich SE-SNe and to SN 2010md, but with lower luminosity. They have previously been the subject of a study as part of a Zwicky Transient Facility (ZTF; Bellm et al. 2019) sample of SLSNe (Yan et al. 2020).

(i) SN 2019cri/ZTF19aanijpu/ATLAS19gnt/Gaia19cpo/PS20axc was discovered by ZTF on 2019-03-25 07:03:21. It was classified as a Type Ic-7 on 2019-04-23 08:47:39 (Prentice et al. 2019e).

(ii) SN 2019dwa/ZTF19aarfyvc/Gaia19bxj was discovered by ZTF on 2019-04-10 07:42:31. It was classified as a Type Ic supernova on 2019-05-16 20:05:46 (Fremling, Dugas \& Sharma 2019).

(iii) SN 2019hge/ZTF19aawfbtgATLAS19och/Gaia19est/PS19elv was another ZTF discovery from 2019-05-31 11:11:30. It was classified as a peculiar SN IIb on 2019-08-23 11:25:41 (Prentice et al. 2019a), and later as a SLSN-I (Dahiwale, Dugas \& Fremling 2019).

(iv) Finally, SN 2019unb/ZTF19acgjpgh/ATLAS19bari/Gaia19fbu /PS19isr was discovered in the ZTF public stream and reported to the Transient Name Server ${ }^{1}$ (TNS) by the Trinity College Dublin transient group on 2019-10-20 12:28:30 (Prentice et al. 2019c). It was classified as a peculiar Type II (Prentice et al. 2019d) and later an SLSN-I (Dahiwale \& Fremling 2020).

\section{DATA COLLECTION AND REDUCTION}

The SNe, shown in context in Fig. 1, were observed with the Liverpool Telescope (LT; Steele et al. 2004). Optical imaging was obtained with the IO:O camera and spectra with the SPectrograph for the Rapid Acquisition of Transients (SPRAT; Piascik et al. 2014). Optical photometry was reduced with a custom PYTHON script utilising PYRAF, which was then calibrated to Sloan Digital Sky Survey (SDSS; Ahn et al. 2014) stars in the respective field. The SPRAT spectra were reduced and calibrated using the LT:SPRAT pipeline (Barnsley, Smith \& Steele 2012) and a custom PYTHON script.

Three of the objects were observed as part of the advanced Public ESO Spectroscopic Survey of Transients (ePESSTO+, for an overview of PESSTO, see Smartt et al. 2015). These are SNe 2019cri, 2019hge, and 2019unb. For the aforementioned objects, spectra were obtained using ESO Faint Object Spectrograph and Camera (v.2; EFOSC2; Buzzoni et al. 1984) on-board the ESO New Technology Telescope. These spectra were reduced through standard pipelines. $^{2}$

We were able to obtain additional spectra using the Intermediatedispersion Spectrograph and Imaging System (ISIS) mounted on the 4.2-m William Herschel Telescope (WHT). This was reduced using a custom IRAF pipeline.

\footnotetext{
${ }^{1}$ https://www.wis-tns.org/

${ }^{2}$ https://github.com/svalenti/pessto
} 

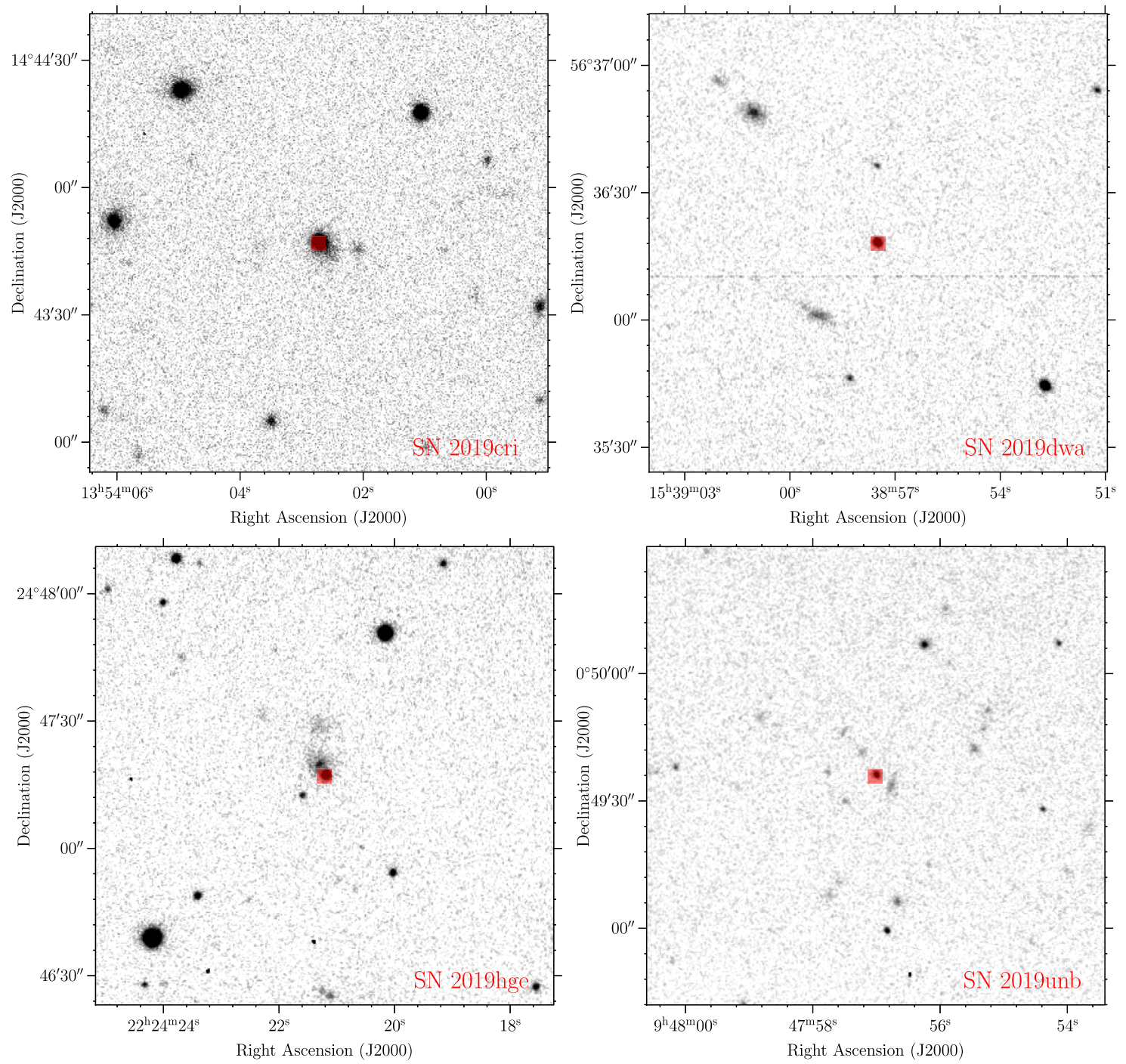

Figure 1. LT $r$-band images of the SN 2019cri (upper left-hand), SN 2019dwa (upper right-hand), SN 2019hge (lower left-hand), and SN 2019unb (lower right-hand) as observed in their respective fields. Owing to the distances involved, the host galaxies are small and dim, and at the location of each transient.

Finally, all proprietary data will be made public via the Weizmann Interactive Supernova Data Repository (WISeREP) ${ }^{3}$

\subsubsection{Distance and extinction}

The distance modulus $\mu$ for each SN was calculated using its redshift, and cosmological parameters from the 9-yr Wilkinson Microwave Anisotropy Probe; $\mathrm{H}_{0}=69.32 \mathrm{~km} \mathrm{~s}^{-1} \mathrm{Mpc}^{-1}, \Omega_{m}=0.286, \Omega_{\Lambda}=$ 0.714 (Hinshaw et al. 2013). Reddening within the Milky Way, $E(B$ $-V)_{\mathrm{MW}}$, were provided by the dust maps of Schlafly \& Finkbeiner (2011). The spectra of each object were checked for evidence of absorption by local dust via $\mathrm{Na}$ I $\mathrm{D}$ absorption lines but no indication was found, thus, we take intrinsic reddening $E(B-V)_{\text {host }}$ for all objects to be negligible. Relevant values are given in Table 1. All data were corrected for $E(B-V)_{\mathrm{Mw}}$ using $R_{V}=3.1$ and the extinction law of Cardelli, Clayton \& Mathis (1989).

\subsection{Host galaxies}

The spectral energy distributions (SEDs) of the host galaxies were modelled with the software package PROSPECTOR ${ }^{4}$ version 0.3 (Leja et al. 2017). We assumed a linear-exponential star formation history, the Chabrier (2003) IMF, the Calzetti et al. (2000) attenuation model, and the Byler et al. (2017) model for the ionized gas contribution. The priors were set as described in Schulze et al. (2021). The results are shown in Fig. 2.

In order to build the SEDs, we retrieved science-ready coadded images from the Galaxy Evolution Explorer (GALEX) general release 6/7 (Martin et al. 2005), the SDSS DR 9 (SDSS; Ahn et al. 2012), the Panoramic Survey Telescope and Rapid Response System (PanSTARRS, PS1) DR1 (Chambers et al. 2016), the Two Micron All Sky Survey (2MASS; Skrutskie et al. 2006), and pre-processed WISE images (Wright et al. 2010) from the unWISE archive (Lang 2014). ${ }^{5}$ The unWISE images are based on the public WISE data and include images from the ongoing NEOWISE-Reactivation mission

${ }^{4}$ https://github.com/bd-j/prospector

${ }^{5} \mathrm{http}: / /$ unwise.me 
Table 1. Redshift, extinction, and distance of each object. Host extinction is assumed to be negligible.

\begin{tabular}{lccccccc}
\hline SN & Type & $\alpha(\mathrm{J} 2000)$ & $\delta(\mathrm{J} 2000)$ & $z$ & $\begin{array}{c}E(B-V)_{\mathrm{MW}} \\
(\mathrm{mag})\end{array}$ & $\begin{array}{c}\text { Comments } \\
(\mathrm{mag})\end{array}$ \\
\hline 2019cri & Ic-7 (peculiar) & $13: 54: 02.720$ & $+14: 43: 46.96$ & 0.041 & 36.31 & 0.02 & Redshift from host H $\alpha$ \\
2019dwa & Ic-7 (peculiar) & $15: 38: 57.480$ & $+56: 36: 18.18$ & 0.076 & 37.71 & 0.01 & Redshift from host H $\alpha$ \\
2019hge & Ib-pec/SLSN-Ib & $22: 24: 21.210$ & $+24: 47: 17.12$ & 0.087 & 38.01 & 0.06 & Redshift from Yan et al. (2020) \\
2019unb & IIb-pec/SLSN-IIb & $09: 47: 57.010$ & $+00: 49: 35.94$ & 0.064 & 37.31 & 0.11 & Redshift from Yan et al. (2020) \\
\hline
\end{tabular}
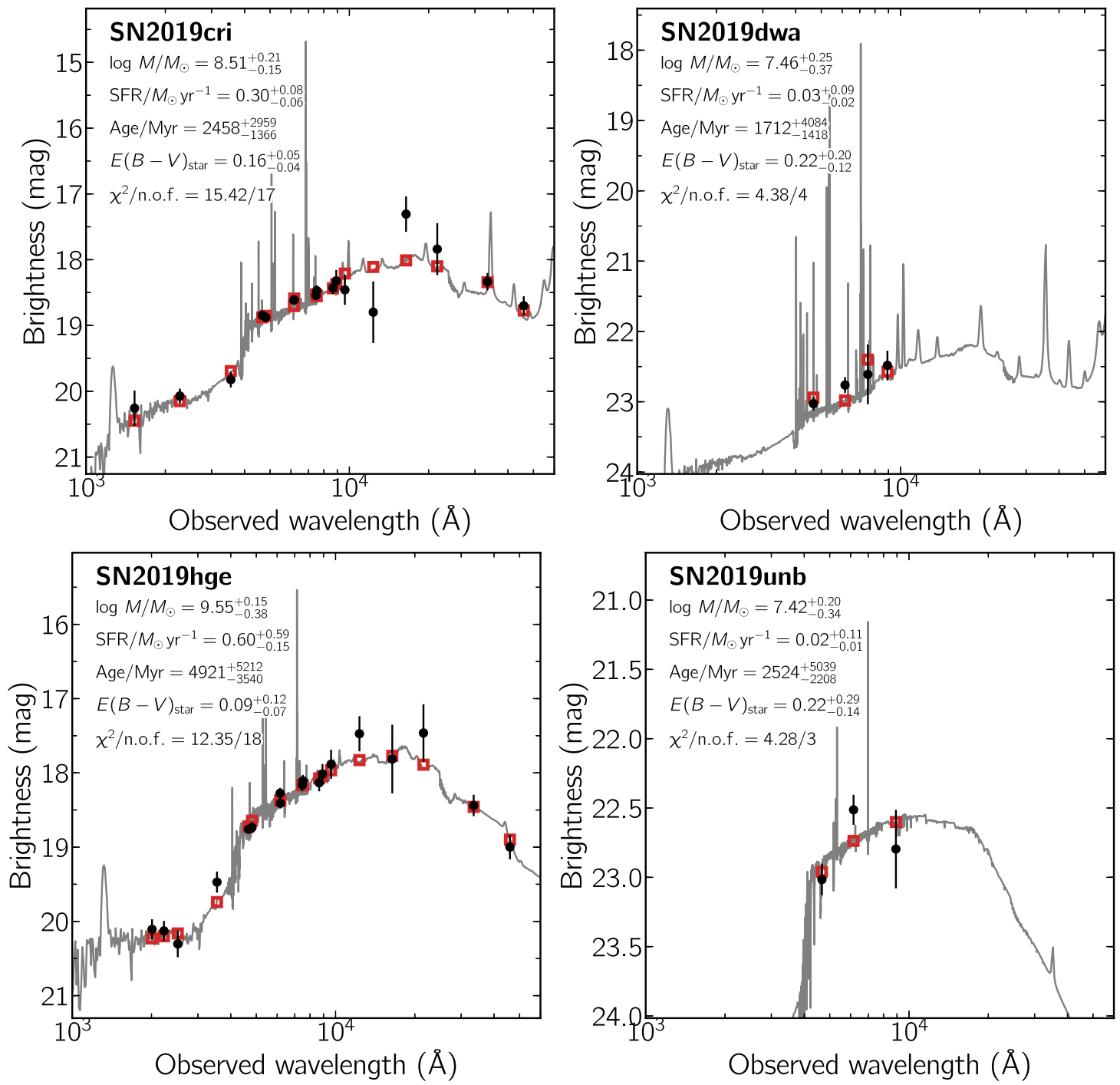

Figure 2. The SED of the host galaxies (detections $\bullet$; model predicted magnitudes $\square$ ). The solid line displays the best-fitting model of the SED. The fitting parameters are shown in the upper left-hand corner. The abbreviation 'n.o.f.' stands for numbers of filters.

R3 (Mainzer et al. 2014; Meisner, Lang \& Schlegel 2017). We also retrieved deeper optical images from the DESI Legacy Imaging Surveys (Legacy Surveys, LS; Dey et al. 2019) DR7 for SN 2019dwa and SN 2019unb. Co-added grz images of the hosts from The Dark Energy Camera Legacy Survey (DECaLS) obtained through the Legacy Survey can be seen in Fig. 3.

The field of SN2019hge was observed with the UV/optical (UVOT; Roming et al. 2005) on-board the Neil Gehrels Swift Observatory (Gehrels et al. 2004). We use the data, after the SN faded, to measure the brightness of the host in the UV filters. The brightness in the UVOT filters was measured with UVOT-specific tools in the HEASOFT ${ }^{6}$ version 6.26.1. Source counts were extracted from the images using a region of 6.8 arcsec. The background was estimated using a circular region with a radius of 39 arcsec close to the $\mathrm{SN}$ position. The count rates were obtained from the images using the Swift tool UVOTSOURCE. They were converted to magnitudes using the UVOT calibration file from 2020 September. All magnitudes were transformed into the AB system using Breeveld et al. (2011).

${ }^{6}$ https://heasarc.gsfc.nasa.gov/docs/software/heasoft/ 

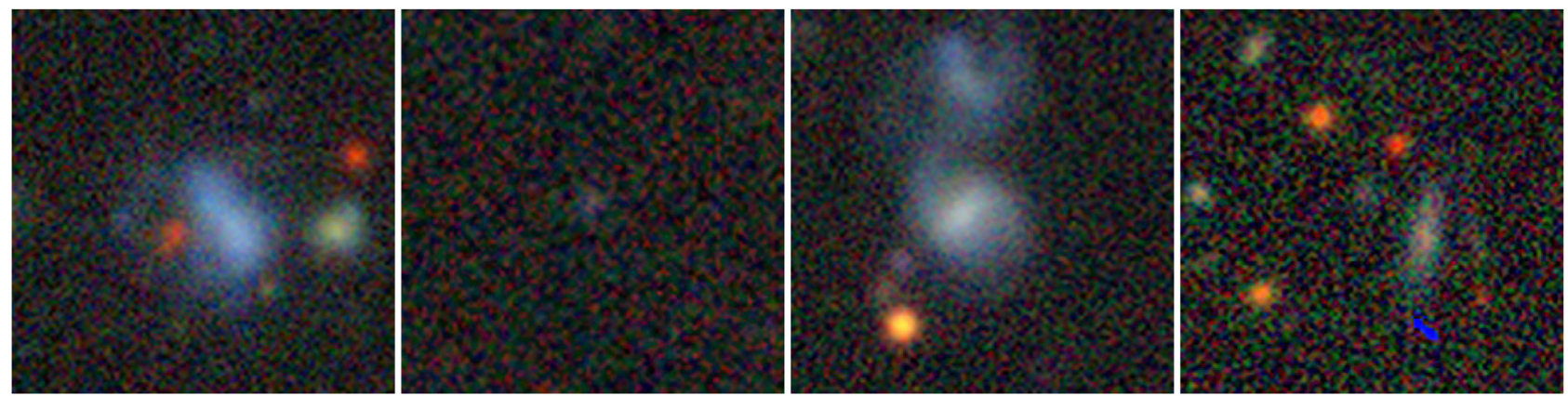

Figure 3. grz cut-outs from The DECaLS. Left-hand panel: the host of SN 2019cri. Centre left-hand panel: the host of SN 2019dwa. Centre right-hand panel: the host of SN 2019hge. Right-hand panel: the host of SN 2019unb. The morphological shape of the hosts of SNe 2019cri and 2019hge provide evidence of recent interaction.

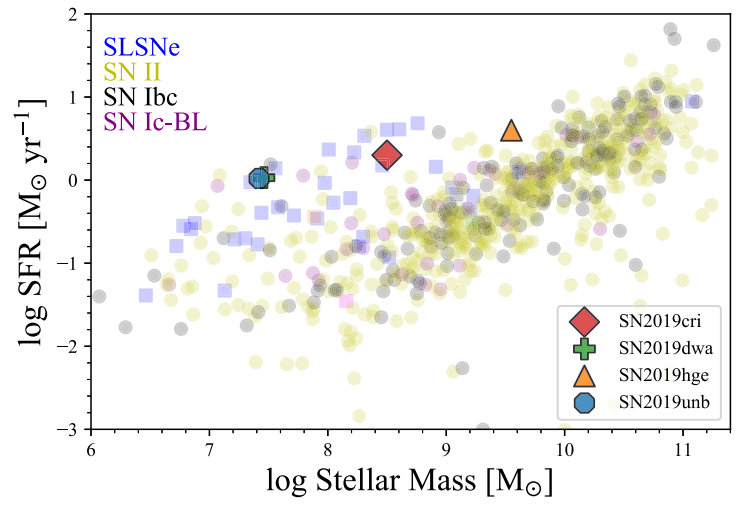

Figure 4. The position of the host of the four $\mathrm{SNe}$ in comparison with the PTF sample of Schulze et al. (2020a). While most of the host galaxies have high star formation and relatively low mass, the host of SN2019hge stands out as being the most massive of the four, and comparable to regular CC-SNe.

We used the software package LAMBDAR (Lambda Adaptive MultiBand Deblending Algorithm in R; Wright et al. 2016), which is based on a software package written by Bourne et al. (2012) and tools presented in Schulze et al. (2021), to measure the brightness of the host galaxy. The brightness in the Legacy Surveys images were measured with the aperture-photometry tool presented by Schulze et al. (2018). ${ }^{7}$

Fig. 4 shows how these host galaxies compare with the hosts of the PTF sample of supernovae in star formation rate/stellar mass space. It can be seen that the hosts of SNe 2019cri, 2019dwa, and 2019unb have typically higher star formation rates than is usual for the hosts of 'normal' CC-SNe, and are consistent with the hosts of SLSNe. In terms of stellar mass, the host of SN 2019hge has the largest and is comparable with the typical hosts of CC-SNe. Finally, by inspecting the host galaxy morphology in the Legacy Survey images, we can see that the hosts of SN 2019cri and SN 2019hge have undergone, or are still undergoing, interaction with another galaxy, Fig. 3.

\section{PHOTOMETRY}

\subsection{Light curves}

Fig. 5 shows the multicolour light curves for each object. Also plotted are the epochs of spectroscopic observations. For both SN 2019hge

${ }^{7}$ https://github.com/steveschulze/Photometry and SN 2019dwa, the unusual nature of the events were only publicly reported quite late in the evolution of each object, so our observations began post-maximum. The presence of the public ZTF light curves are testament to the importance of public data release from large surveys, as without this data, neither of these objects would have been identified as being unusual or been subject to a follow-up campaign.

From Fig. 5, there are two obvious defining features for each event. The first is the long-lived rise to $r$-band maximum light. This is at least $65 \mathrm{~d}$ for SN 2019cri, 35d for SN 2019dwa, $60 \mathrm{~d}$ for SN 2019hge, and $70 \mathrm{~d}$ for SN 2019unb. The second, are the variations in brightness prominently seen in SN 2019cri, SN 2019hge, and SN 2019unb. These variations are highly reminiscent of another recent event, SN2019stc, which was also identified as a transitional event between SLSNe and normal SE-SNe (Gomez et al. 2021).

\subsubsection{Light-curve morphology}

The brightness variations are unlike the typical evolution of SE-SNe, although they are seen in SLSNe. If we consider SN 2019cri, the light curves rise to an initial peak after $\sim 60 \mathrm{~d}$, before they decay but then level off between $80-100 \mathrm{~d}$. The light curves then undergo another period of decay, but this last little beyond $150 \mathrm{~d}$ because the final observations at $\sim 240 \mathrm{~d}$ show little change from the observations almost $100 \mathrm{~d}$ prior.

SN 2019dwa shows the least variation in brightness, however, around $65-70 \mathrm{~d}$, some variation is seen in $r$ that is present in both the LT and the ZTF photometry. A curious aspect of SN 2019dwa's light curve, aside from the long rise, is that after peak the light curve decays for nearly $100 \mathrm{~d}$ without ever settling on a ${ }^{56} \mathrm{Co}$ tail. This will be discussed further in relation to other objects in Section 3.1.2.

Next, we consider the complex light curves of SN2019hge. The transient rises to a peak over $60-70 \mathrm{~d}$. Around $30 \mathrm{~d}$, the light curve appears to 'stall' in its rise before continuing up to maximum light. At around $70 \mathrm{~d}$, the light curve drops 0.7 and 1 mag over about a week for $r$ and $g$, respectively. The ZTF light curve then begins a slow rise again, at which point our photometric and spectroscopic observations start. The rise lasts $12 \mathrm{~d}$ before decaying further, at a slightly slower rate than previously. At about $125 \mathrm{~d}$, the decay ceases and another rise is seen in the ZTF $g$ and $r$ photometry. This decays again before settling on a relatively flat tail. At this point, the object was no longer visible during the night, it was not later recovered.

Finally, SN 2019unb evolves in a similar way to SN 2019hge. There is a rise to a plateau over $30 \mathrm{~d}$, the plateau is seen in the optical bands but not in $u$, which decays from the first observation. After $\sim 55 \mathrm{~d}$, the griz light curves rise again until they reach maximum 

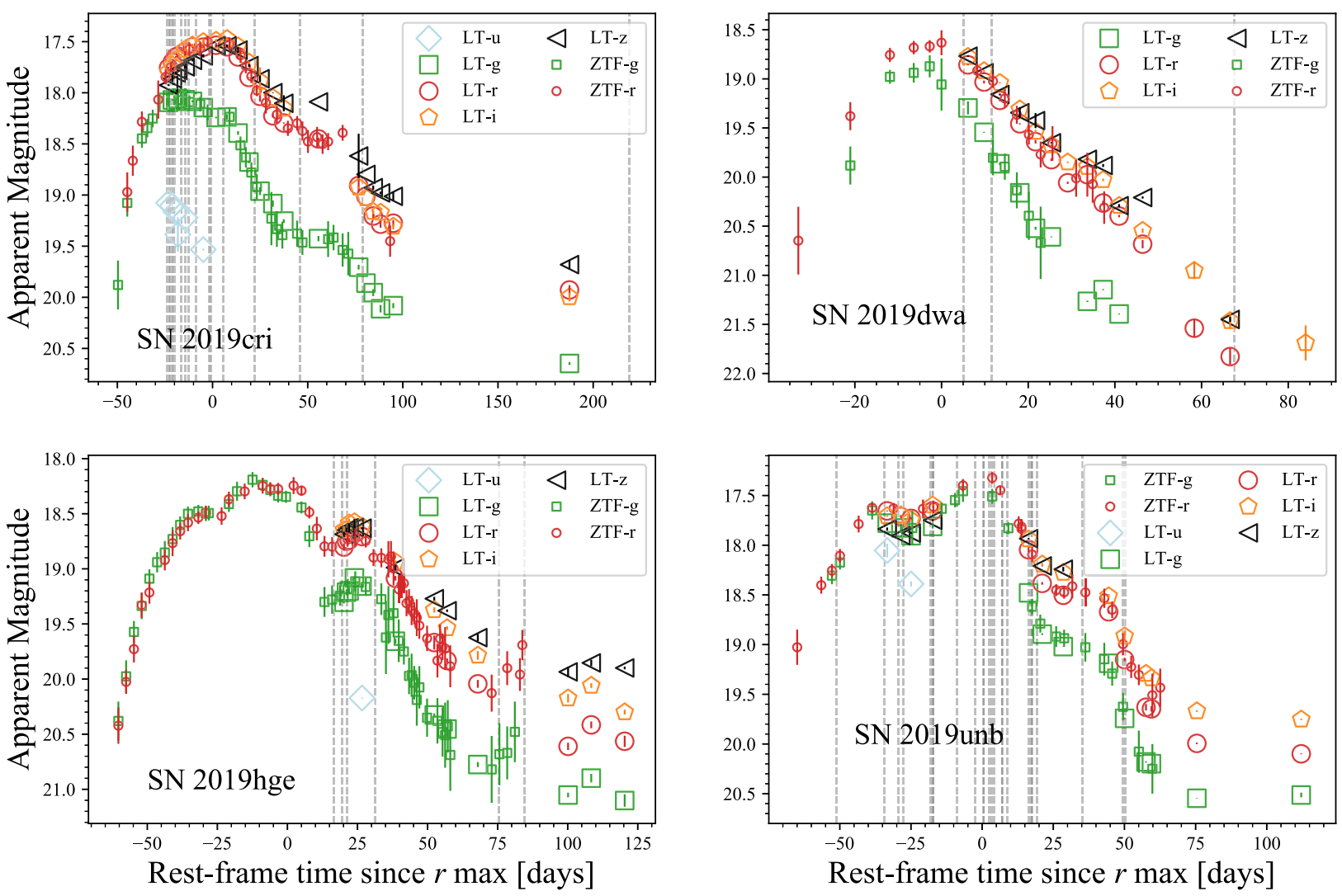

Figure 5. The multicolour LT light curves of the four SNe featured here. Included in the figure is the public ZTF P60 photometry. Top left-hand panel: SN 2019cri. Top right-hand panel: SN 2019dwa. Lower left-hand panel: SN 2019hge. Lower right-hand panel: SN 2019unb. Grey dashed lines denote epochs of spectroscopic observations.

light at $70 \mathrm{~d}$. The light curves then decay until around $95 \mathrm{~d}$, with $g$ decaying more rapidly than $r$ as per SN 2019hge. There is then a period with much slower decay before the light curves return to a rapid decay between $120-150 \mathrm{~d}$. At this point, the light curve is sparsely sampled but shows a very flat evolution. The photometry is not contaminated by the host and was the transient was clearly detected in the images on these days.

\subsubsection{Absolute r-band light curves}

To estimate the absolute $r$-band light curves of the $\mathrm{SNe}$, the relevant light curve was corrected for $E(B-V)_{\mathrm{MW}}$ as per Table 1 then a $K$-correction was applied. These were derived from the spectra and assumed to be constant (the same as the calculated value nearest in time) outside of the epoch of spectroscopic observations. While this does miss the time-dependence of the $K$-correction at these phases, the overall corrections are relatively small $(\sim|0.1| \mathrm{mag})$ and applied at phases away from maximum light. Most affected is SN 2019dwa, where the $g$-band K-corrections approach 0.3 mag and the spectroscopic time-series only covers a few weeks. Finally, the absolute magnitude was found by subtracting the distance modulus $\mu$ from the corrected apparent magnitude. The peak $M_{r}$ and $M_{g}$ are given in Table 2.

Fig. 6 shows these light curves against a selection of SE-SNe and SLSNe. The four transients have peak $M_{r}$ between -19 and $-20.1 \mathrm{mag}$, which places them in between the space of the luminous SNe Ibc and the sub-luminous SLSNe. SN2019dwa is the least
Table 2. Intrinsic peak magnitude and full width at half maximum light for $M_{g}$ and $M_{r}$.

\begin{tabular}{lcccc}
\hline SN & $\begin{array}{c}M_{g} \\
(\mathrm{mag})\end{array}$ & $\begin{array}{c}\text { Width } \\
(\mathrm{d})\end{array}$ & $\begin{array}{c}M_{r} \\
(\mathrm{mag})\end{array}$ & $\begin{array}{c}\text { Width } \\
(\mathrm{d})\end{array}$ \\
\hline 2019cri & $-19.02 \pm 0.02$ & 64 & $-19.45 \pm 0.03$ & 69 \\
2019dwa & $-19.0 \pm 0.1$ & 29 & $-19.0 \pm 0.1$ & 39 \\
2019hge & $-19.98 \pm 0.06$ & 57 & $-19.86 \pm 0.07$ & 83 \\
2019unb & $-20.2 \pm 0.1$ & 64 & $-20.21 \pm 0.05$ & 67 \\
\hline
\end{tabular}

luminous of the four in $M_{r}$ and also has the narrowest light curve, as defined by the length of time the light curve is more luminous than half its peak luminosity (full width half maximum; FWHM).

Brighter still is SN 2019cri, SN 2019hge, and then SN 2019unb. As is discussed in Section 4, SNe 2019cri and 2019dwa are spectroscopically similar to normal SNe Ic, while SNe 2019hge and 2019unb are similar to $\mathrm{SNe} \mathrm{Ib} / \mathrm{IIb}$ at later phases but at early phases have a SLSN-like blue spectrum. The well-studied SLSN SN 2015bn (Nicholl et al. 2016a) peaks at nearly two orders of magnitude greater than our four objects, which are closer to the luminosity distribution of SE-SNe.

It was previously noted that SN 2019dwa has an unusual decline that does not reach a ${ }^{56} \mathrm{Co}$ tail by at least $70 \mathrm{~d}$ after maximum. Comparison with the $\mathrm{SNe}$ Ibc shows how unusual this is, as the decaying light curve of SN2019dwa passes through the position of the late linear tails of many objects that are dimmer at peak magnitude. 


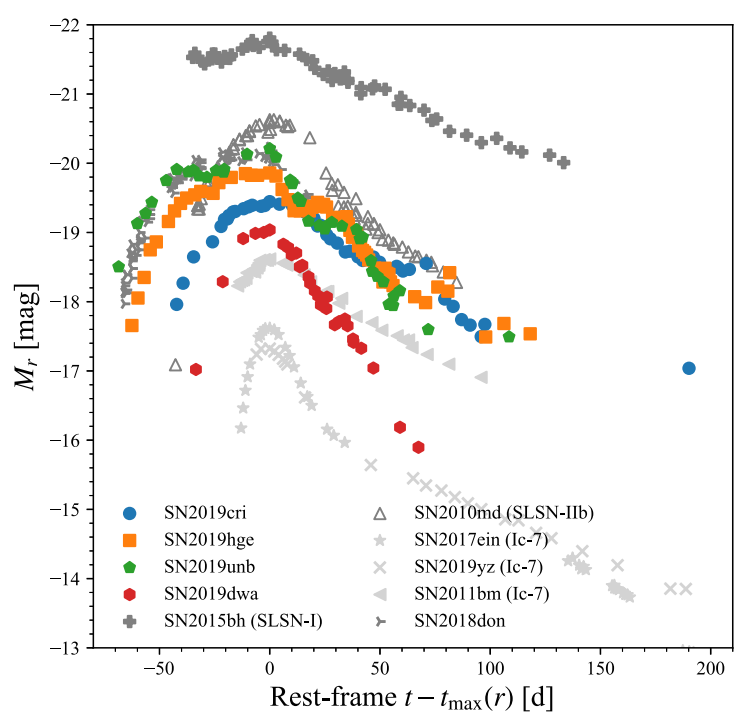

Figure 6. The absolute $r$-band magnitude of the four SNe. For comparison are a sample of SNe Ic-7 - SNe 2011bm (Valenti et al. 2011), 2017ein (Teffs et al. 2021), and 2019yz. SNe Ibc are generally found in the region bounded by these objects. Also included is a representative SLSN-I (SN 2015bn Nicholl et al. 2016a), as well as SLSN-IIb 2010md (Quimby et al. 2018) and luminous SN 2018don. The four SNe presented here lay between the two supernova groups, in a region known for the lack of H-poor CC-SNe. Despite the classification of SNe 2019hge and 2019unb as SLSNe, only SN 2019unb has $M_{r}<-20 \mathrm{mag}$, and this only fleetingly.

Prior to the discovery of SN 2019cri, the Type Ic-7 SN $2011 \mathrm{bm}$ (Valenti et al. 2012) displayed the broadest known light curve for a spectroscopically normal SNe Ic. Fig. 6 demonstrates that SN 2019cri is a considerably broader and more luminous an event. The spectroscopic similarity between these SNe Ic-7 shown in the figure is discussed in Section 4.1. SN2019cri is also similar photometrically to the luminous H/He-poor SN 2018don (Lunnan et al. 2020) and transitional object SN 2012aa (Roy et al. 2016), all display a long rise followed by a decline, a period of levelling off and then a secondary decline. Spectroscopically however, neither SN 2018don or SN 2012aa display the strong narrow absorption of SN 2019cri.

\subsection{Colour curves}

The $g-r$ colour curves derived from the dereddened and K-corrected $g$ and $r$ photometry are shown in Fig. 7. For comparison are a sample of SNe Ibc from Prentice et al. (2019b) and a sample of SLSNe. Owing to the difference in temporal evolution between the $\mathrm{SNe}$ Ibc and the comparison object, the time-scales of the SNe Ibc are multiplied by a factor of three.

SNe 2019cri and 2019dwa, which are both He-poor, follow the scaled evolution of the SNe Ibc. They both initially evolve slowly to the red, with SN 2019cri reaching a turnover at the same time as the scaled SE-SN colour curves. This is not apparent in the colour curve of SN 2019dwa and reflects that its light curves are never observed to settle on a late linear tail. In SE-SNe, the epoch of this turnover defines the early nebular phase when emission lines start appearing in the spectra, and this is also the case for SN 2019cri. The two SNe are bluer than the average for the SE-SNe, a slightly lower scaling would better fit the colour curve of SN 2019dwa but it does serve to show that the scaled evolution of the objects are similar. The

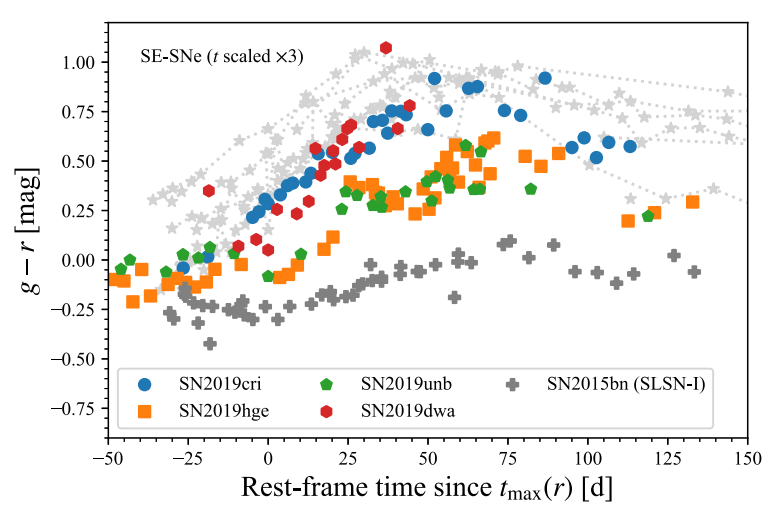

Figure 7. $g-r$ colour curves for the four transients, and for comparison the colour curves of a sample of SE-SNe (light grey) and SLSN 2015bn (dark grey). The four SNe and SN 2015bn have had K-corrections applied.

relationship between colour curves and reddening is well established (see Drout et al. 2011; Stritzinger et al. 2018) and would suggest that the assumption of negligible $E(B-V)_{\text {host }}$ is valid.

SNe 2019hge and 2019unb have very similar colour curves, especially in terms of the shape. They both start with a constant colour for some $50 \mathrm{~d}$ before evolving to the red, they then both take a series of blue-red-blue turn at similar times. They are bluer than the SE-SNe, but redder than the SLSNe but their colour curve shape is not similar to either, so no scaling will match the other objects in the same way as for SNe 2019cri and 2019hge.

\section{SPECTROSCOPY}

The previous section demonstrated that these SNe have peculiar lightcurve evolution compared with SE-SNe and SLSNe. In this section, the spectroscopic observations of each object will be discussed in turn after we first establish each object's similarity to a reference object.

\subsection{Comparison with other objects}

The classification and parameters of the SNe in question depend upon similarity with known transients and their properties. This applies in particular in the line identification. Comparisons with various SE$\mathrm{SNe}$ are shown in Fig. 8.

Starting with the simplest case first, that of SN 2019cri. It can be seen in Fig. 8 that this transient has very similar optical spectra to Type Ic-7, which are the narrow line H/He-poor SE-SNe (Prentice \& Mazzali 2017). SNe in this sub-class include SN 2007gr (Hunter et al. 2009), which is shown for comparison, as well as SN $2011 \mathrm{bm}$ (Valenti et al. 2012), SN 2017ein (Teffs et al. 2021), SN 2005az, SN 2014L, and SN 2019yz. ${ }^{8}$ These SNe are characterized by having the lowest photospheric velocities of $\mathrm{SNe}$ Ic but the largest range of light-curve morphologies and luminosities, which suggests that objects in this spectroscopic sub-type can have a large range of ejecta masses $M_{\mathrm{ej}}$ and nickel masses $M_{\mathrm{Ni}}$.

Next, we find that SN 2019dwa matches the spectra of several SNe Ic and $\mathrm{SNe} \mathrm{Ib}$, with a preference for the former. Our spectra are all more than a week after $r$-band maximum light, and SE-SNe at this phase become increasingly difficult to classify as their spectra tend to appear similar. The absence of strong He I lines at 6678 and 7065

${ }^{8}$ Data for this object is presented in conjunction with this work. 


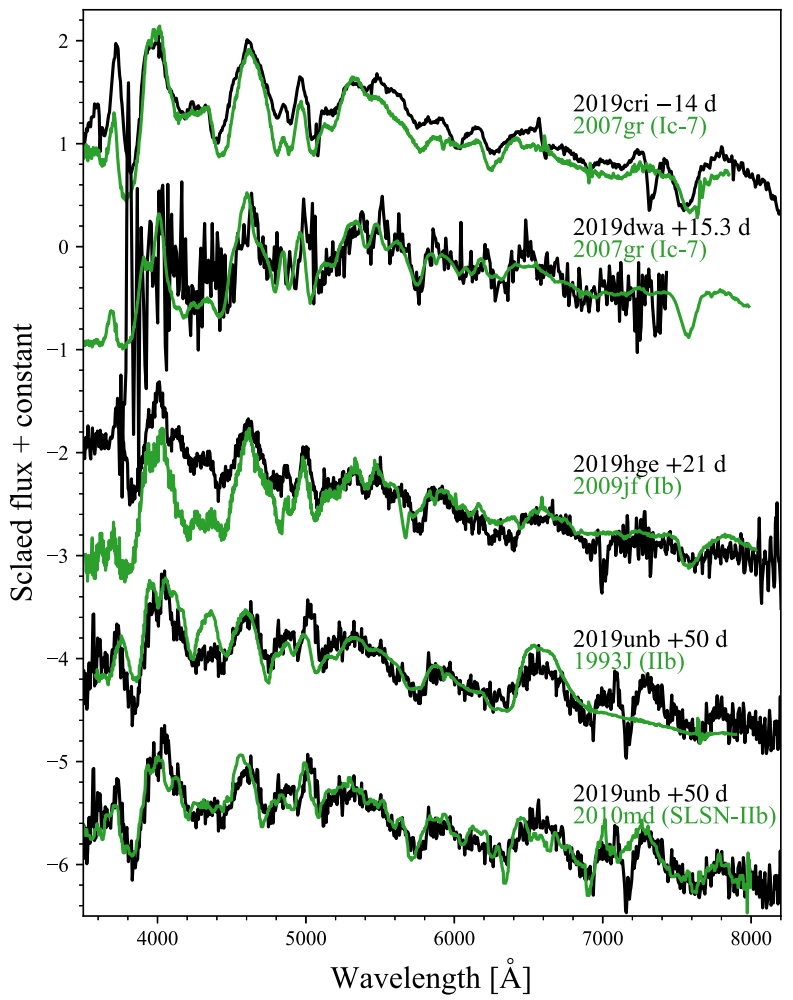

Figure 8. Spectroscopic comparisons between the four objects and spectra of SE-SNe in the photospheric phase. SNe 2019cri and 2019dwa match Ic-7 object, shown for comparison is SN 2007gr (Hunter et al. 2009). SN 2019hge is matched against the Type Ib 2009jf, and SN 2019unb against Type IIb SN 1993J and SLSN-IIb 2010md. Additional matches can be found between these objects and most SNe Ib and $\mathrm{H}$-weak SNe IIb. Note that the comparison spectra have been shifted slightly in velocity space to match the line velocities of the target objects.

$\AA$ would support that this is an SNe Ic. There is an absorption at $5800 \AA$, but this is likely to be due to Na I D rather than He I $\lambda 5876$. The comparison with Ic-7 SN 2007gr shows that this line is present in a similar strength in SNe Ic.

Finding comparison objects for SN2019hge and SN2019unb is considerably more difficult, as these are genuinely unusual objects spectroscopically. We attempted to match the early spectra, but no match with publicly available data was adequate. We did find matches to SE-SNe at the later phases of each object; SN Ib 2009jf (Valenti et al. 2011) for SN 2019hge, and Type IIb SN 1993J (Matheson et al. 2000) for SN 2019unb, these comparisons are shown in Fig. 8. As can be seen, the features of the transients overlap, although SN 2009jf is redder than the others. The SN 2009jf and SN 1993J spectra are not plotted in the rest frame, a redshift was artificially introduced to match the line velocities seen in the other two objects. Fundamentally, this is unimportant however, as line velocities vary from object to object and this shift can be replicated by comparing spectra with small shifts in velocity space (See Mazzali et al. 2017). SN 2019unb also matched the SLSN-IIb 2010md (Quimby et al. 2018; Shivvers et al. 2019) during its later phases, but it may also be said that SN 2010md matched He-rich SE-SNe in its later phases, and its peak luminosity was similar to that of SN 2019unb. The failure to find spectroscopically similar objects at earlier phases, and the later similarity to $\mathrm{SN} \mathrm{Ib/IIb,} \mathrm{led} \mathrm{to} \mathrm{an} \mathrm{exploratory} \mathrm{attempt} \mathrm{to} \mathrm{replicate}$ the early spectra of SNe 2019hge and 2019unb by convolving the pre-maximum spectra of several SE-SNe and a thermal continuum.

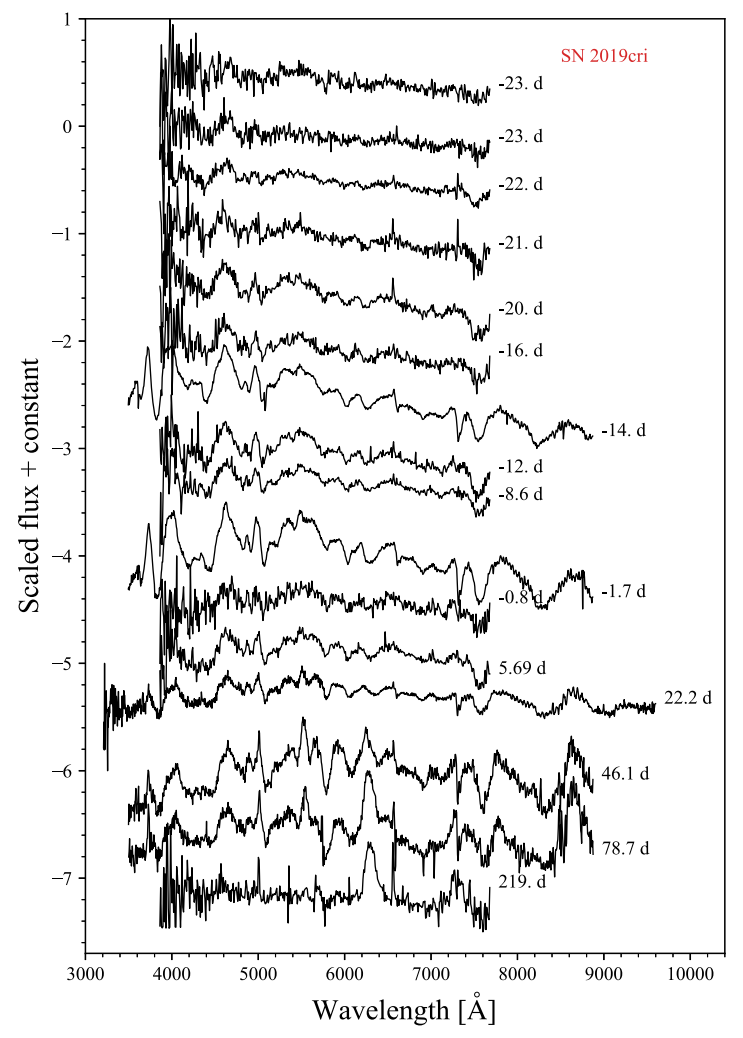

Figure 9. The spectroscopic sequence of SN 2019cri as observed by the LT and NTT. Despite the slow photospheric evolution, the SN enters the early nebular phase two months after maximum light, which is consistent with the time-scales for normal SNe Ic. All phases are relative to the time of $r$-band maximum light.

This led to some success in replicating features in the earlier spectra that could be identified as $\mathrm{Ca}$ II $\mathrm{H} \& \mathrm{~K}, \mathrm{Fe}$ II, He I of $\mathrm{Na}$ I D, and $\mathrm{H} \alpha$ or Si II. This is investigated in more detail in Section 5.3.

\subsubsection{Classification}

As has been demonstrated, SN 2019cri is spectroscopically similar to $\mathrm{SNe}$ Ic-7 and we retain this classification. Likewise, from limited spectroscopy of SN 2019dwa, we find more of a match to narrow line $\mathrm{SNe}$ Ic-7 rather than to $\mathrm{SNe} \mathrm{Ib}$.

There exists no neat box in which to place SN2019hge and SN 2019unb, they could be classified as SN Ib-pec and SN IIb-pec, respectively, where 'pec' is short for 'peculiar', or SLSN-Ib/IIb. It has been shown that these objects, while luminous, are not clearly at SLSN luminosities. Therefore, they may represent a transitional form of event that in different circumstances could lead to either a normal SN IIb or a SLSN.

\subsection{The spectra of SN 2019cri}

Fig. 9 shows the spectroscopic observations of SN 2019cri. As previously discussed, the spectroscopic evolution is virtually identical to that of SNe Ic-7. The most significant difference is an absorption feature around $6100 \AA$. This is not usually seen in SE-SNe and it may be OI $\lambda 6158$. The maximum light spectrum is modelled in Section 5.1 in order to investigate this feature.

The [O I] $\lambda \lambda 6300,6364$ emission line is present in the spectra at $+46.1 \mathrm{~d}$, which would be a typical time-scale for normal SE-SNe. 


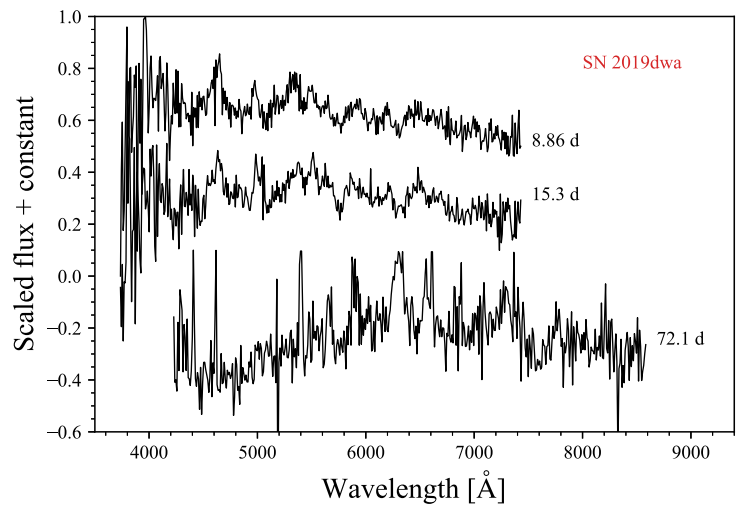

Figure 10. Spectroscopic observations of SN 2019dwa. The earliest two spectra are from the LT. The final spectrum is from the WHT, and is affected by poor flux calibration. The important aspect of this spectrum is the presence of the [O I] $\lambda \lambda 6300,6364$ and [Ca II] $\lambda \lambda 7291,7323$ lines. There is also a narrow $\mathrm{H} \alpha$ line present from the host galaxy. These confirm the core-collapse origin of this object and set the redshift as $z=0.076$.

This contrasts with the object's unusual photometric evolution, which would lead one to expect a later transition into the early nebular phase. By $+78.7 \mathrm{~d}$, the $[\mathrm{O} \mathrm{I}] \lambda \lambda 6300,6364$ line is the dominant feature in the spectrum, also present is the [Ca II] $\lambda \lambda 7291,7323$ emission line. The observation coincides with the time that the light curve begins to fall rapidly again. At $+219 \mathrm{~d}$, the spectrum is entirely nebular. Assuming that the entire emission line at $\sim 6300 \AA$ is from one [O I] $\lambda \lambda 6300,6364$ doublet, the FWHM of the [O I] $\lambda 6300$ component is $5000 \pm 200 \mathrm{~km} \mathrm{~s}^{-1}$. This is at the very low end of the FWHM velocity distribution for SNe (Prentice et al. submitted), and shows that the relatively low photospheric velocities are also seen in the nebular phase. Finally, taking the flux ratio of [Ca II] $\lambda \lambda 7291,7323 /[\mathrm{OI}]$ $\lambda \lambda 6300,6364$ gives $\sim 0.8$.

It is important to point out that there is no indication of $\mathrm{H}$ or He emission lines in the photospheric spectra, and there are no signatures within the spectra that mark the changes in the light curve. Spectroscopically, SN 2019cri is the same as any other Ic-7.

\subsection{The spectra of SN 2019dwa}

SN 2019dwa was too far North to be observed by ePESSTO+, so we obtained just three spectra for this object; two shortly after one another about a week after $r$ maximum. Fig. 10 shows that they are of low signal to noise but provide enough information to say that the spectra are not $\mathrm{H}$ or He rich and there are no signs of emission lines. The earliest spectra, taken just after $r$-band maximum, match well with that of SN Ic-7.

Two spectra were taken of the transient at $+72.1 \mathrm{~d}$ using the WHT. The combined spectrum is noisy, and we reject any features that are not present in both spectra, but we can see that the dominant emission at this time is from [O I] $\lambda \lambda 6300,6364$, also present is [Ca II] $\lambda \lambda 7291,7323$ and $\mathrm{Na}$ I D. A full width half-maximum measurement of the [O I] $\lambda 6300$ component to the doublet is $5000 \pm 300 \mathrm{~km} \mathrm{~s}^{-1}$. Like SN 2019cri, this is consistent with measurements of SE-SNe (Taubenberger et al. 2009) but is lower than the average. We also detect $\mathrm{H} \alpha$ in emission, and an emission line at $5670 \AA$, which could be $[\mathrm{N} \mathrm{II}] \lambda 5680$. The presence of these lines provides a redshift of $z=$ $0.076 \pm 0.02$ for the transient. The $\mathrm{H} \alpha$ line is noisy but thin, which suggests that it is emission from the host galaxy, this is discussed further in Section 2.1 where this interpretation is at odds with the

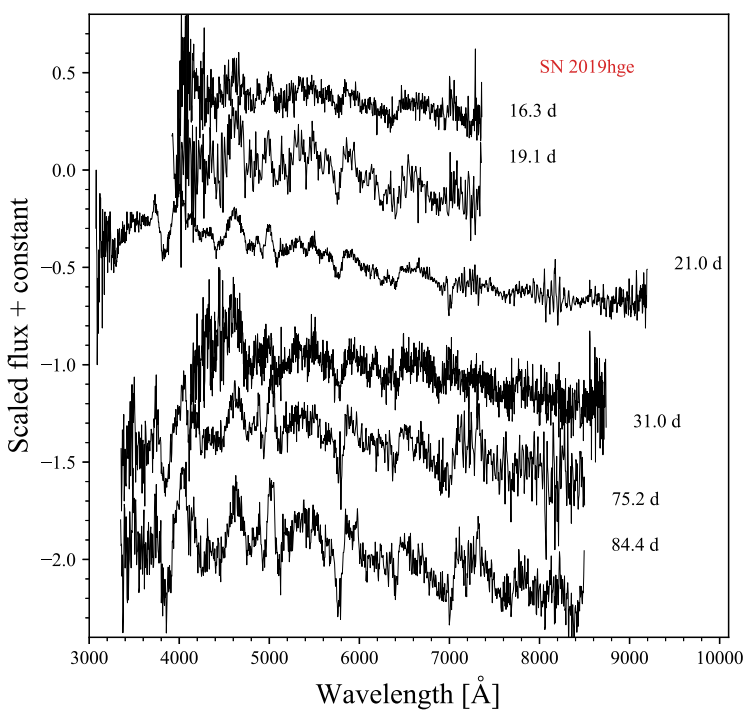

Figure 11. The spectra of SN 2019hge, phases are in the rest-frame and relative to $r$ maximum.

photometry of the pre-explosion site. If this is the case, then this line may be weak emission from interaction.

\subsection{The spectra of SN 2019hge}

Our observations of SN2019hge only began after the secondary maximum, these a presented in Fig. 11. At these epochs, the spectra are typically SN Ib-like, with features from Fe II $\lambda, \operatorname{Mg} I \lambda, \mathrm{Ca} I I$ $\mathrm{H} \& \mathrm{~K}$, in the blue, He I $\lambda \lambda 5876,6676,7065$, in the mid part of the spectrum, and O I $\lambda 7774$ and the Ca II NIR triplet in the red. As with the previously discussed objects, there is no evidence for external influences on the spectra via IIn/Ibn like narrow emission lines that could explain the light-curve variation.

The ZTF classification spectrum from MJD 58665.41 (Dahiwale et al. 2019) is $30 \mathrm{~d}$ after discovery ( $\sim 48$ rest-frame days prior to our first spectrum) and coincides with the brief flattening in the light curves during the rise. This spectrum is blue, as to be expected from the colours. The colour curves also suggest that this spectrum changes little in terms of continuum slope for much of the rise. This spectrum does not display the characteristic 'w' feature between 4000-5000 that is associated with O II and is common in SLSN-I.

\subsection{The spectra of SN 2019unb}

Unlike SN 2019hge, SN 2019unb was recognized early as an unusual event. Our first series of spectra (Fig. 12) from -50 to $+7 \mathrm{~d}$ are defined by a hot continuum with absorption present from $\mathrm{Ca}$ II $\mathrm{H} \& \mathrm{~K}, \mathrm{He}$ I $\lambda 5876$ and/or Na I D, and also possibly some weak Fegroup features between 4000-5000. . As with SN 2019hge, these are revealed through a simple convolution between the spectra of SE-SNe and a thermal continuum, but it is also acknowledged that the temperature would suggest different ionization regimes for the various elements and it is possible that such features may be a chance alignment. The spectra do not show the numerous $\mathrm{C}$ II and $\mathrm{O}$ II lines common to SLSNe, and as with the previous objects, we find nothing that evolves to trace the variations in the light curves and we find no obvious emission lines. The maximum light spectrum is modelled in Section 5.3. 


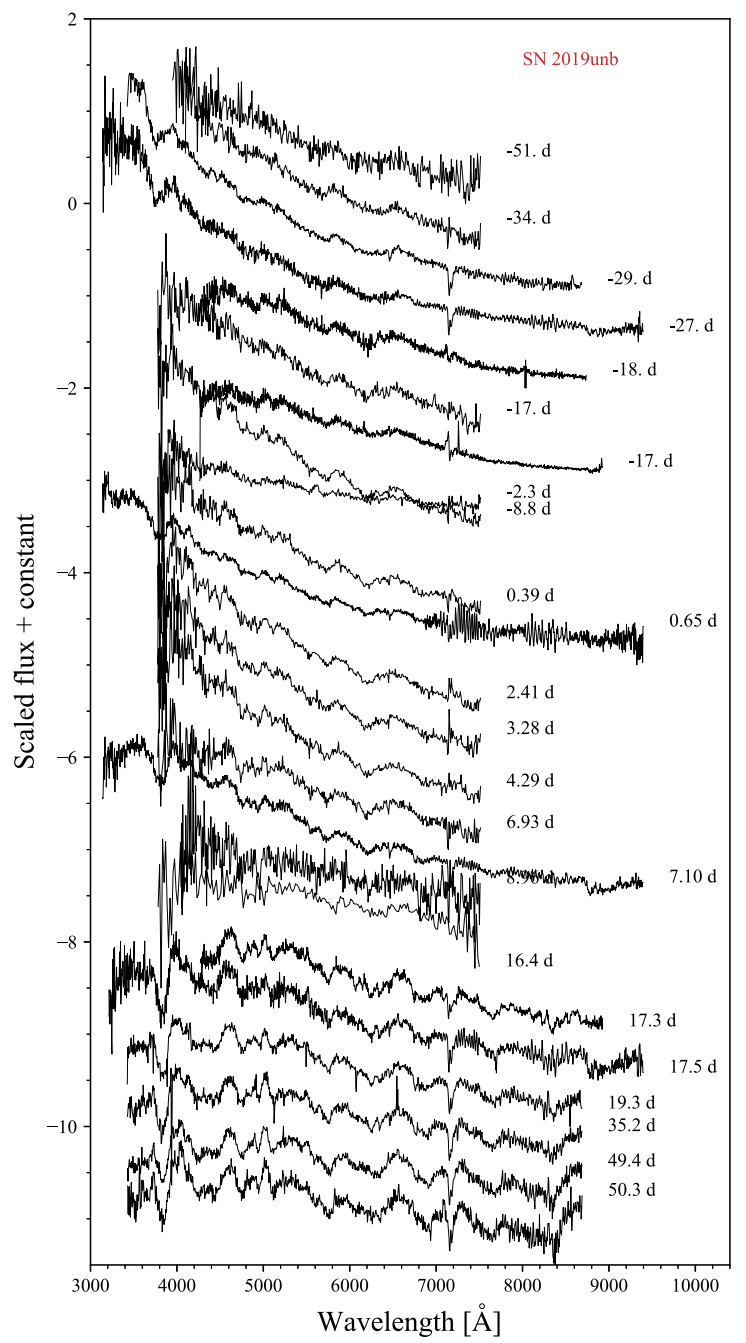

Figure 12. The spectroscopic sequence of SN 2019unb. Phases are relative to $r$ maximum.

Throughout its evolution, SN2019unb displays a broad bump around $6563 \AA$, which becomes more prominent over time and which may be $\mathrm{H} \alpha$. There is no measurable absorption component to this line however, but it does suggest the presence of $\mathrm{H}$ in the ejecta. This would also support the presence of He and thus the comparison with $\mathrm{SNe} \mathrm{Ib} / \mathrm{IIb}$.

\subsection{Line velocities}

To further characterize the properties of the $\mathrm{SNe}$, photospheric phase line velocities were measured from the absorption minima of elements common to such transients. Fig. 13 shows the measurements for Fe II $\lambda 5169$, Si II $\lambda 6355$, Ca II (due to limited spectroscopic wavelength, this is measured from both the NIR feature and H\&K), and He I $\lambda 5876$ where possible. Also presented for comparison are a small sample of SNe Ib/Ilb (Prentice et al. 2019b) and a sample of SNe Ic-7, including SN $2011 \mathrm{bm}$.

\subsubsection{Fe II $\lambda 5169$}

In terms of Fe II velocity, all four transients have significantly lower velocities that any of the comparison objects. SN 2019cri shows evolution over time, from $\sim 8000$ to $\sim 5000 \mathrm{~km} \mathrm{~s}^{-1}$ and evolves on a similar time-scale to $\mathrm{SN} 2011 \mathrm{bm}$. If the velocity curve is scaled by $15 / 65$, which represents the ratio of rise times for a normal SNe Ic compared to SN 2019cri, then the curve shapes are similar between it and the SNe Ic-7, but with SN 2019cri being $\sim 2500 \mathrm{~km} \mathrm{~s}^{-1}$ slower. That the velocity decreases until around optical maximum light and then levels off, is characteristic to all SNe Ic. Only two measurements can be made of SN 2019dwa and both suggest that it also has low velocities at this phase. SN 2019hge was observed well after peak, and displays an Fe II velocity commensurate with $\mathrm{SNe}$ $\mathrm{Ib} / \mathrm{IIb} \sim 6000 \mathrm{~km} \mathrm{~s}^{-1}$. There is a reduction in this velocity over time to $\sim 3000 \mathrm{~km} \mathrm{~s}^{-1}$ at $+80 \mathrm{~d}$. SN 2019 unb has the lowest maximum light Fe II velocity of all at $v \sim 4500 \mathrm{~km} \mathrm{~s}^{-1}$. The Fe II lines are not strong however, so measurement is made by considering Fe II $\lambda \lambda 4924,5018$ as well. The measured velocities represent the only set of features that lined up with the strong series of Fe II lines between 4000 and $5000 \AA$. Comparison with SN 2009jf in Fig. 8 shows that the 4000-5000 regions are similar at later times. In SN 2009jf, which is one of the comparison objects in Fig. 13, the Fe II velocities are easily traceable.

\subsubsection{Si II $\lambda 6355$}

This line is commonly measured in SNe Ic, it can be seen in Fig. 13 that SNe 2019cri and 2019dwa have lower velocities than the SNe Ic7. The maximum measured velocity for SN $2019 \mathrm{cri}$ is $\sim 6000 \mathrm{~km} \mathrm{~s}^{-1}$ compared with $10000-12000 \mathrm{~km} \mathrm{~s}^{-1}$ for the comparison objects. Both SN 2019cri and SN 2019dwa show a post-maximum velocity of $\sim 3000 \mathrm{~km} \mathrm{~s}^{-1}$, approximately $4000 \mathrm{~km} \mathrm{~s}^{-1}$ slower than the SNe Ic.

\subsubsection{CaII}

The velocity measurements of Ca II NIR and Ca II H\&K show the most similarity to the comparison SNe. SNe 2019hge and 2019unb both follow the same track as the SNe Ib/IIb, as does SN 2019cri although this is a few $1000 \mathrm{~km} \mathrm{~s}^{-1}$ slower than the SNe Ic. Ca II is often found to evolve with similar velocities to Fe II (Prentice et al. 2019b), this is likely due to both being produced by primordial elements in the ejecta and so both trace the photosphere. This causes some tension for SN 2019unb with it's standard Ca II velocity but very low Fe II velocity.

\subsubsection{Hе I $\lambda 5876$}

Finally, a comparison of the He I measurements for SNe 2019hge and 2019 unb shows that they have lower line velocities compared to the bulk of these objects. However, scaling for rise times shows that the observed evolution of both are very similar to the $\mathrm{SNe} \mathrm{Ib} / \mathrm{IIb}$. This line is often a blend of Na I and He I $\lambda 5876$ in SNe Ib, and in Section 5.3, it is shown that modelling can replicate this particular feature well with just Na I D. The strength of the feature pre-peak is comparable to $\mathrm{SNe} \mathrm{Ib}$ rather than $\mathrm{SNe}$ Ic however, which would suggest that $\mathrm{He} \mathrm{I}$ is present at this time.

\subsubsection{Summary}

The line velocities in the photospheric phase of these objects are generally lower than most, if not all of the comparison objects. The velocity curves evolve slowly, which partially reflects the long photometric evolution of these objects. However, the relative line velocities do not scale directly with the relative light-curve timescales, for example, although SN 2019unb is approximately 4-5 time broader than an $\mathrm{SN} \mathrm{Ib}$, its velocities are only approximately half by 

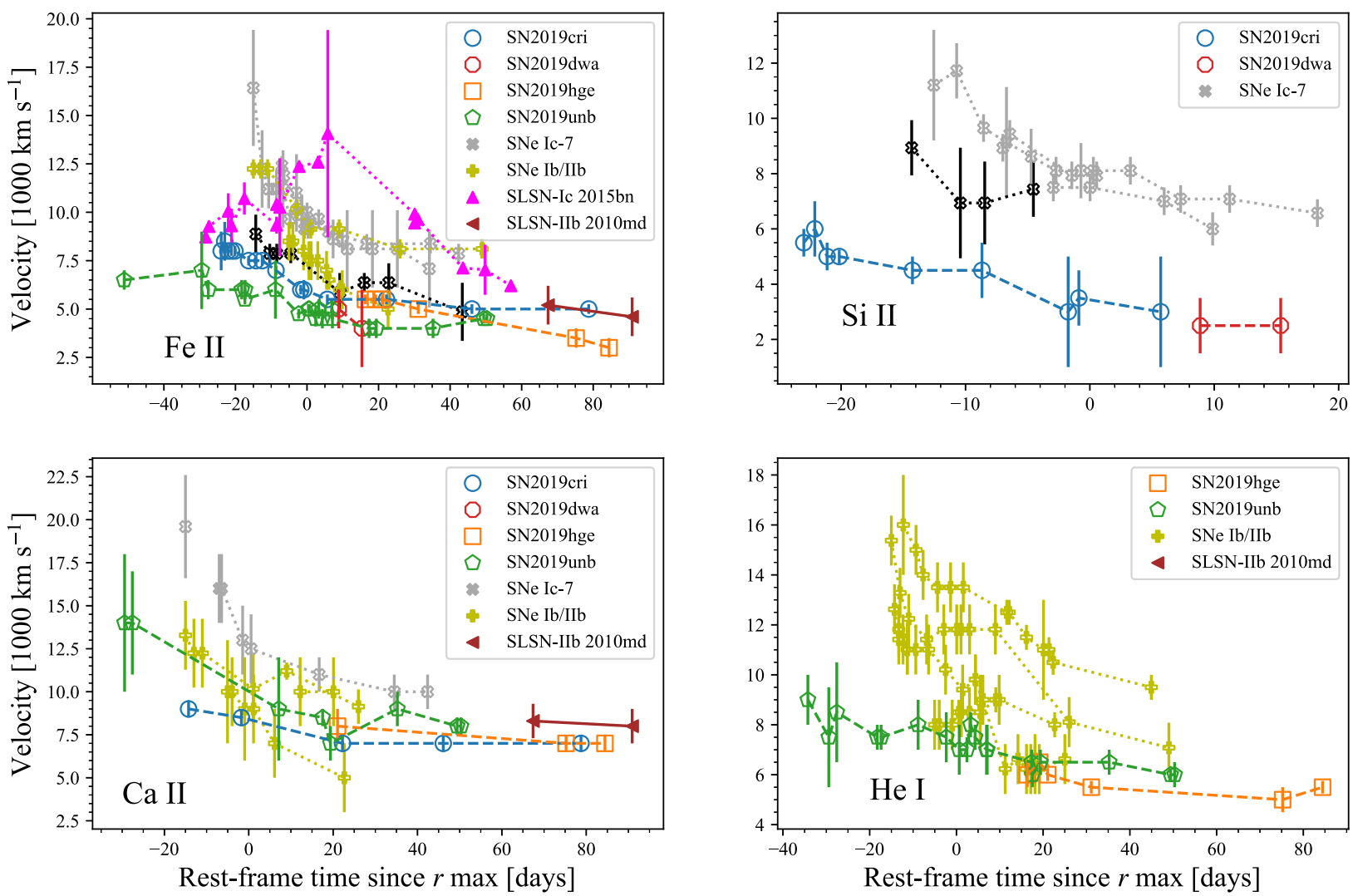

Figure 13. The line velocities measured as a function of time in comparison with SNe Ic-7 (dark grey, SN 2011bm emphasised in black), SNe Ib/IIb (yellow), SLSN-Ic 2015bn (mageneta; Liu, Modjaz \& Bianco 2017b) and SLSN-IIb (brown; Inserra et al. 2013). Top left-hand: Fe II $\lambda 5169$. Top right-hand: Si II $\lambda 6355$. Lower left-hand: Ca II. Lower right-hand: He I $\lambda 5876$.

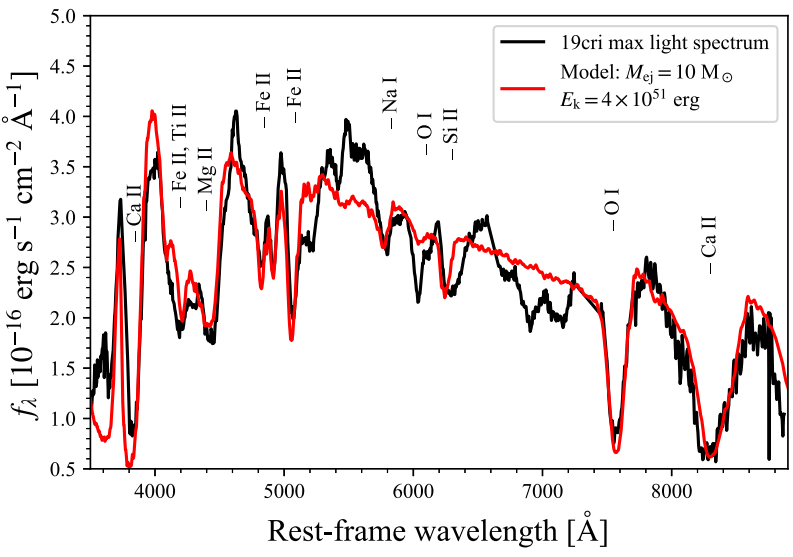

Figure 14. Snapshot modelling of SN 2019cri using a density profile of $10 \mathrm{M}_{\odot}$ and $E_{\mathrm{k}}=4 \times 10^{51} \mathrm{erg}$.

comparison. As with normal $\mathrm{SNe}$, the velocities do not increase at any point. However, this is not well-constrained as even changes of $10 \mathrm{~s}$ of percent are below the resolution of the spectra. Slow velocity evolution can be produced by low specific kinetic energies and/or steep gradients in the ejecta density profile.

\section{SNAPSHOT SPECTRAL MODELLING}

Owing to the similarity of SN2019cri and SN 2019dwa to SNe Ic, and the lack of obvious $\mathrm{H}$ and $\mathrm{He}$ in the maximum/pre-maximum

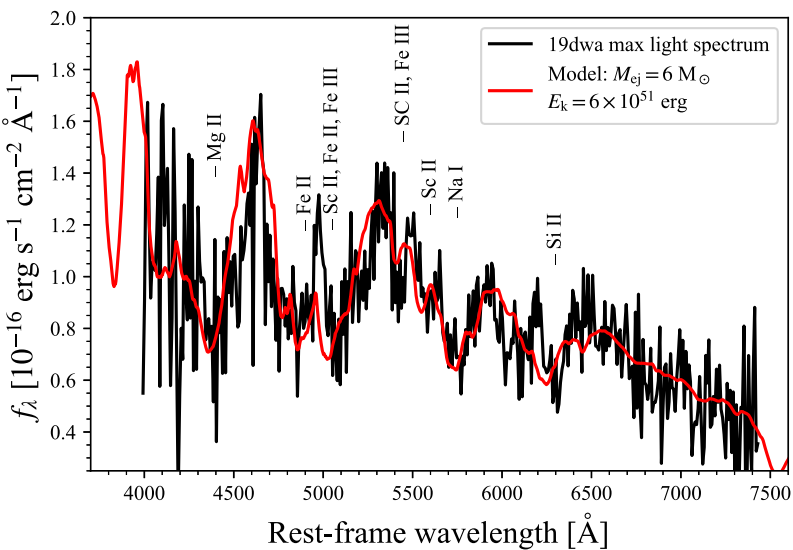

Figure 15. Modelling of SN 2019dwa using a density profile of $6 \mathrm{M}_{\odot}$ and $E_{\mathrm{k}}=6 \times 10^{51} \mathrm{erg}$.

spectra of SN 2019unb, we took the opportunity to make a 'snapshot' model of the maximum light spectrum for each object using a wellestablished one-dimensional code for the synthesis of SN spectra (Mazzali \& Lucy 1993; Lucy 1999; Mazzali 2000). The code, based on the Monte Carlo method, uses the Schuster-Schwarzschild approximation and enforces radiative equilibrium in the expanding $\mathrm{SN}$ ejecta. It requires as input a density/abundance distribution with radius (Stehle et al. 2005), as well as an emergent luminosity and the epoch since explosion of the spectrum. Photons emitted at the photosphere are allowed to interact with the gas in the ejecta via 


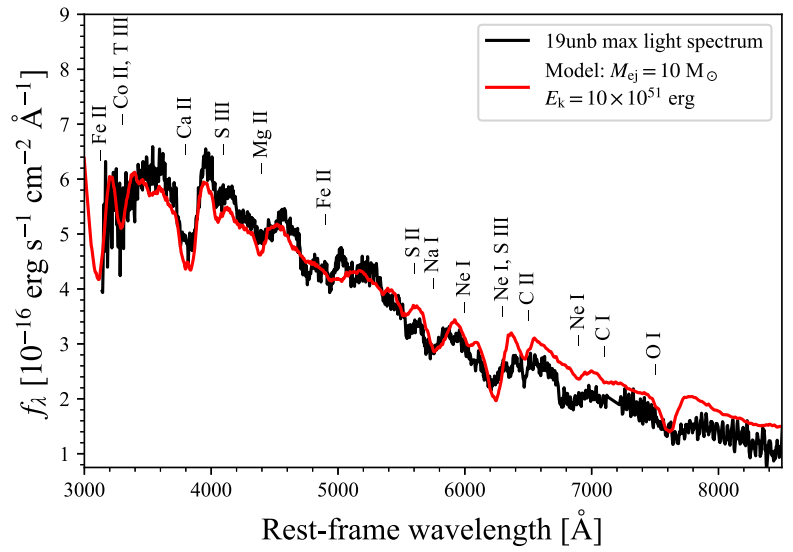

Figure 16. Modelling of SN 2019 unb using a density profile of $10 \mathrm{M}_{\odot}$ and $E_{\mathrm{k}}=10 \times 10^{51} \mathrm{erg}$. Time since explosion is $80 \mathrm{~d}$. This snapshot recreates most of the features seen in the observed spectrum.

absorption processes, which can be followed by re-emission in different lines, thereby implementing both fluorescence and reverse fluorescence processes, and to scatter off free electrons, which increases their residence time in the ejecta. Excitation and ionization are computed using the nebular approximation, which is appropriate in these low density environments (Pauldrach et al. 1996).

This code has been applied numerous times on stripped-envelope transients (e.g. Mazzali et al. 2002; Sauer et al. 2006; Mazzali et al. 2017; Ashall et al. 2019; Pian et al. 2020; Teffs et al. 2021), including SLSNe (Mazzali et al. 2016), and was used for a parameter study in relation to extremely energetic SNe Ic (Ashall \& Mazzali 2020). For our models, we needed to create ad-hoc density profiles. Given that the SNe we analysed can be classified as Ic-7, which suggests a similar specific kinetic energy $E_{\mathrm{k}} / M_{\mathrm{ej}}$ but that their light curves indicate a possible range of masses, we started with model CO21 (Nomoto et al. 1994), which was developed to model the spectra and light curves of Ic-6 SN 1994I. The initial abundances were based upon the work of Sauer et al. (2006). Next, we set suitable luminosities $L$ and photospheric velocities $v_{\mathrm{ph}}$ in order to match the flux level and line velocity of the spectrum. We rescaled this model in $M_{\mathrm{ej}}$ and $E_{\mathrm{k}}$ using the rescaling equations of of Hachinger et al. (2009). We used the assumption that objects with similar spectra have similar $E_{\mathrm{k}} / M_{\mathrm{ej}}$ (e.g. Mazzali et al. 2013; Teffs et al. 2021). This was then used to constrain $E_{\mathrm{k}}$ to $M_{\mathrm{ej}}$, with adjustments made to fit the line widths. The ejecta mass was found by fixing the time of the spectrum since explosion $t-t_{\exp }$ and then iterating the mass scaling until viable fits were found with a dilution factor of the model $w$ is between 0.4 and 0.6. Finally, we adjusted the elemental abundances in order to optimize the fit to the observed spectra.

The process of determining optimal parameters is iterative, but when convergence is achieved it likely to point to the correct values given the model that has been used. While individual parameters can have uncertainties ranging from 5 to 25 per cent, when they are taken all together in a 'best-fitting model', the uncertainty is likely to be much smaller, of the order of 5-10 percent (Ashall \& Mazzali 2020).

\subsection{Modelling the maximum light spectrum of SN $2019 \mathrm{cri}$}

The redshift and extinction corrected maximum light spectrum, in relation to the $r$-band, is dated 2019-05-14 and was observed by the NTT. A density profile with $M_{\mathrm{ej}}=10 \mathrm{M}_{\odot}$ and $E_{\mathrm{k}}=4$ foe (foe $=$ $1 \times 10^{51} \mathrm{erg}$ ), and a specific kinetic energy of 0.4 [foe/ $\left.\mathrm{M}_{\odot}\right]$ was found to give appropriate model spectra at the required time $t$. This compares favourably with the $0.56\left[\right.$ foe/ $\left.\mathrm{M}_{\odot}\right]$ found for SN 2017ein (Teffs et al. 2021). The time since explosion $t-t_{\exp }=65 \mathrm{~d}$, with a photospheric velocity $v_{\mathrm{ph}}=5700 \mathrm{~km} \mathrm{~s}^{-1}$. The abundance at this time assuming a single shell is 50 per cent $O, 29$ per cent $C, 20$ per cent $\mathrm{Ne}$, with the remaining 1 per cent split between (in decreasing order) $\mathrm{Mg}, \mathrm{Si}, \mathrm{S}, \mathrm{Na},{ }^{56} \mathrm{Ni},{ }^{56} \mathrm{Fe}$, Ti, and $\mathrm{Ca}$.

The kinetic energy was constrained through the width of the lines, in particular the Fe II and $\mathrm{Mg}$ I absorptions around $5000 \AA$, which are distinct and are characteristic of SNe Ic-7. A larger kinetic energy leads to broader lines that blend together. This constraint on the kinetic energy is limited however, as it is sampling the lower velocity material. If the earlier spectra had displayed broader lines then this would have required a shallower density profile at these epochs which results in an increase in kinetic energy without an equivalent increase in ejecta mass, this then would increase the specific kinetic energy (Mazzali et al. 2013). The photospheric velocity at this epoch is consistent with the measured line velocities in the spectra (see Section 4.6).

Despite this, a point of concern remains; the density profile used to give this snapshot is designed to produce the standard light curve of a supernova and could not replicate the variations seen in the postpeak light curves of SN 2019cri. Part of the problem is that energy deposition from the ${ }^{56} \mathrm{Ni}$ decay chain decreases in a predictable manner over time, and that the light-curve variations are achromatic in the optical. There are no indicators in the spectra either to suggest this is a recombination effect, as in the secondary bump in the $r$-band light curves of SNe Ia. Speculatively, this may then suggest that the luminosity variation is due to an increase in continuum flux and may arise from thermalization of energy emitted from a source in the interior of the ejecta.

\subsection{The $+8 \mathrm{~d}$ spectrum of SN 2019dwa}

For SN 2019dwa, the $+8.7 \mathrm{~d}$ spectrum is used. It has unfortunately low $\mathrm{S} / \mathrm{N}$ and presents a limited wavelength range of approximately $4000-7400 \AA$ in the SN rest-frame. The spectrum is still quite blue compared with SE-SNe at this relative age, but shows signs of being evolved, such as deep absorption features and low velocities. A similar process for SN 2019cri was used to find a density profile suitable combination of $M_{\mathrm{ej}}$ and $E_{\mathrm{k}}$. The provisional model has $M_{\mathrm{ej}}=6 \mathrm{M}_{\odot}$ with $E_{\mathrm{k}}=6$ foe, giving $E_{\mathrm{k}} / M_{\mathrm{ej}}=1$ [foe $\left./ \mathrm{M}_{\odot}\right]$, at $t$ $-t_{\exp }=45 \mathrm{~d}$ with $v_{\mathrm{ph}}=3000 \mathrm{~km} \mathrm{~s}^{-1}$. The ions responsible for the features in the range 4000-5300 $\AA$ are mainly $\mathrm{Mg}$ II Fe II and Fe III. In the intermediate region, we find Na I D and Si II $\lambda 6355$, with also the likely detection of Sc II resulting in absorptions, most prominently between $5400-5600 \AA$. Sc is also identified through spectral modelling in the post-maximum spectra of SN 2017ein (Teffs et al. 2021). The abundances used in the one-zone model at this time are 25 per cent $\mathrm{O}, 20$ per cent $\mathrm{Ne}, 15$ per cent $\mathrm{Si}, 15$ per cent $\mathrm{C}$, 10 per cent $\mathrm{Na}, 9$ per cent ${ }^{56} \mathrm{Ni}$, and 1 per cent $\mathrm{Sc}$ and 5 per cent split between $\mathrm{Ca}, \mathrm{Mg},{ }^{56} \mathrm{Fe}$, and $\mathrm{S}$.

\subsection{SN 2019unb at +7.2 d}

The spectrum $+7.2 \mathrm{~d}$ after $r$-band maximum of SN 2019unb is not dominated by $\mathrm{H}$ or $\mathrm{He}$, so the same method can be applied to model this spectrum. Through an iterative process, a density profile with $M_{\mathrm{ej}}=10 \mathrm{M}_{\odot}$ and $E_{\mathrm{k}}=10$ foe was chosen. The time since explosion $t$ $-t_{\text {exp }}=80 \mathrm{~d}$, and photospheric velocity $v_{\text {ph }}=4900 \mathrm{~km} \mathrm{~s}^{-1}$. The low $v_{\text {ph }}$ supports the Fe II velocities that were measured in Section 4.6. The spectra do not extend blueward enough to constrain the flux 
below $3200 \AA$, but the total bolometric luminosity of the model is $2.6 \times 10^{43} \mathrm{erg} \mathrm{s}^{-1}$ (-19.8 mag), comparable to the peak of $M_{r}$ at this time. The ejecta composition is typical for a massive star; $\mathrm{Ca}, \mathrm{C}$, $\mathrm{O}, \mathrm{S}, \mathrm{Si}, \mathrm{Fe}, \mathrm{Na}, \mathrm{Ne}$. In the model, the abundances are 35 per cent $\mathrm{O}, 40$ per cent $\mathrm{C}, 20$ per cent $\mathrm{Ne}$, with the remaining 5 per cent split between $\mathrm{Na}, \mathrm{Si}, \mathrm{S}, \mathrm{Mg},{ }^{56} \mathrm{Fe}$, and ${ }^{56} \mathrm{Ni}$. This leads to an overly strong O I $\lambda 7774$ line in the one shell model. A solution to this would be to place some of the excess mass that is in the $\mathrm{O}$ fraction into an element that is not strong in the optical, which could be He. The presence of an ' $\mathrm{H} \alpha$ bump' in the later spectra would suggest some presence of $\mathrm{H}$, and therefore $\mathrm{He}$, in the ejecta. However, at this phase neither are identified. ${ }^{9}$ For the $\mathrm{H}$ Balmer features this is likely because the photospheric temperature is $\sim 8000 \mathrm{~K}$ and so $\mathrm{H}$ is mostly ionized. Helium, on the other hand, needs to be non-thermally excited by the decay products of the ${ }^{56} \mathrm{Ni}$ decay chain, but at $80 \mathrm{~d}$ most of the ${ }^{56} \mathrm{Ni}$ and it's daughter nuclei have decayed to ${ }^{56} \mathrm{Fe}$.

\section{DISCUSSION}

\subsection{Powering mechanisms}

In addition to the large kinetic energies required, which exceed those for explosion modelling (Müller 2015), the luminous, long-lived, and variable light curves of these objects leads to the question of the powering mechanism. Here, we discuss a few potential sources for powering of these events.

The analytical equations of Arnett (1982) can be used to estimate the mass of ${ }^{56} \mathrm{Ni}$ required to power the respective light curves. This 'back of the envelope' calculation is used purely to illustrate the difficulty in powering these objects with ${ }^{56} \mathrm{Ni}$. If we take the basic approximation that $M_{r}$ approximated the absolute magnitude at bolometric maximum, then we can convert these values to cgs luminosity units. Combining this with the rise time and using the formulation of 'Arnett's Rule' given in Stritzinger \& Leibundgut (2005), we get the following back-of-the-envelope $M_{\mathrm{Ni}}$ estimates of $\sim 1.5 \mathrm{M}_{\odot}$ for $\mathrm{SN} 2019 \mathrm{cri}, \sim 1 \mathrm{M}_{\odot}$ for $\mathrm{SN} 2019 \mathrm{dwa}, \sim 3.5 \mathrm{M}_{\odot}$ for SN 2019hge, and $\sim 4.7 \mathrm{M}_{\odot}$ for SN 2019unb. These should be considered an upper limit, as recent explosion models have suggested that 'Arnett's Rule' could lead to an overestimate of the ${ }^{56} \mathrm{Ni}$ mass (Khatami \& Kasen 2019). In each case, the estimated $M_{\mathrm{Ni}}$ would still constitute a considerable fraction of the ejecta mass. This would lead to SN Ia-like temperatures, line blanketing, and emission dominated by Fe-group lines in the later phases, which is not seen. Instead, the results of the spectral modelling suggests that the amount of Fe group elements is relatively comparable to that in normal corecollapse events. This also rules out possibility of these events being pair-instability $\mathrm{SNe}$, as the ejected mass is far too small.

If, as is most likely, that ${ }^{56} \mathrm{Ni}$ is not the power source behind these objects the we must look to alternative options. An easy way to reach the luminosities seen in these objects along with the variations in the light curves, is to have the ejecta interact with CSM. In this process, a small fraction of the kinetic energy is converted to radiative energy, and because $E_{\mathrm{k}} \sim 10^{51}$ erg for a typical $\mathrm{SN}$, this provides and ample energy reservoir to power the light curves. Under normal circumstances, the effect of ejecta interacting with CSM is prominent spectroscopic narrow emission lines of $\mathrm{H}$ or $\mathrm{He}$, depending on the CSM composition. This respectively leads to Type IIn and Type Ibn

${ }^{9}$ Both $\mathrm{H}$ and He require NLTE treatment, which is beyond the scope of this work. However, while He I $\lambda 5876$ may contribute some small amount to the $\mathrm{Na}$ I feature in the modelled spectrum, $\mathrm{H}$ is entirely absent.
$\mathrm{SNe}$, both of which can reach luminosities greater than the object featured in this work (e.g, Hosseinzadeh et al. 2017; Nyholm et al. 2020). It was noted in Section 4 that none of these objects show emission lines consistent with CSM interaction. We can consider this an external process, as the energy injection occurs on the outside of the ejecta, however interaction has been proposed as a way to power the transitional event PTF11rka without explicit spectroscopic signatures of interaction (Pian et al. 2020).

An internal process would include radioactive powering (e.g. ${ }^{56} \mathrm{Ni}$ and ${ }^{56} \mathrm{Co}$ decay), or some physical process that taps the rotational energy of a compact object. In this latter category, we find black hole accretions and magnetars. Magnetars are rapidly rotating highly magnetized neutron stars. Their energy reservoir and energy injection rate is governed by two parameters; the period $P$ of rotation and the strength of the magnetic field $B$. Magnetars with millisecond spin periods and $B \sim 10^{15}$ Gauss can have a total rotational energy of $>1 \times 10^{52} \mathrm{erg}$ and provide a diverse range of monotonically decreasing energy injection rates. These objects are commonly invoked for SLSNe, as they provide a way to power these objects without requiring tens of solar masses of ejecta and several solar masses of ${ }^{56} \mathrm{Ni}$, which is difficult to synthesize. Although most of the spin-down power must be thermalized in order to explain the high continuum luminosities of SLSNe, possible signatures of magnetar powering are present in the spectrum: at early times, broad $\mathrm{O}$ II lines seen in most SLSNe and some lower luminosity SNe could indicate non-thermal ionization in rapidly expanding ejecta (Mazzali et al. 2016, Parrag et al., submitted), while at nebular phases a central energy source is needed to power a prominent $\mathrm{O}$ I recombination line (Nicholl et al. 2016b, 2019; Jerkstrand et al. 2017). However, the transitional events studied here lack O II lines and we have few nebular phase spectra. Another possible means to test the presence of a central engine is via the evolution of the photospheric velocity. Kasen \& Bildsten (2010) suggested that magnetars may be able to produce cavities at the base of the ejecta equivalent to pulsar winds, with the consequence being that ejecta is pushed from below and accelerated into ejecta above, forming a high density 'wall'. An observational effect of this density spike could be a flat or very slow velocity evolution in spectroscopic lines. However, this density spike may also be an artefact of one-dimensional simulations, and can be washed out by turbulent mixing in higher dimensions (Chen, Woosley \& Sukhbold 2016), but simulations suggest this is energy-dependent (Suzuki \& Maeda 2019, 2021).

The line velocities in the objects here are indeed slow, amongst the slowest evolving seen in stripped CC-SNe. SN 2019cri provides a good comparison because another explanation for low, and slowly evolving velocities is low energy. However, comparison of the line velocity curves in Fig. 13 with SN Ic-7 (equivalent specific kinetic energy) shows that this is not an impediment to having a relatively normal velocity profile.

A central compact object powering these $\mathrm{SNe}$ is an attractive idea. The evidence suggests that the powering source is probably not external unless all four objects were able to hide the CSM interaction below their photosphere. The luminosity variability of such an object, perhaps through accretion, is also a possible way to explain the variations in the light curves without affecting the appearance of the optical spectra.

\subsection{Undulations and their origin}

As observed in Section 3.1, these objects show some degree of undulations in their luminosity evolution. This is reminiscent of what observed in SLSNe I like SN 2015bn (Nicholl et al. 2016a; 


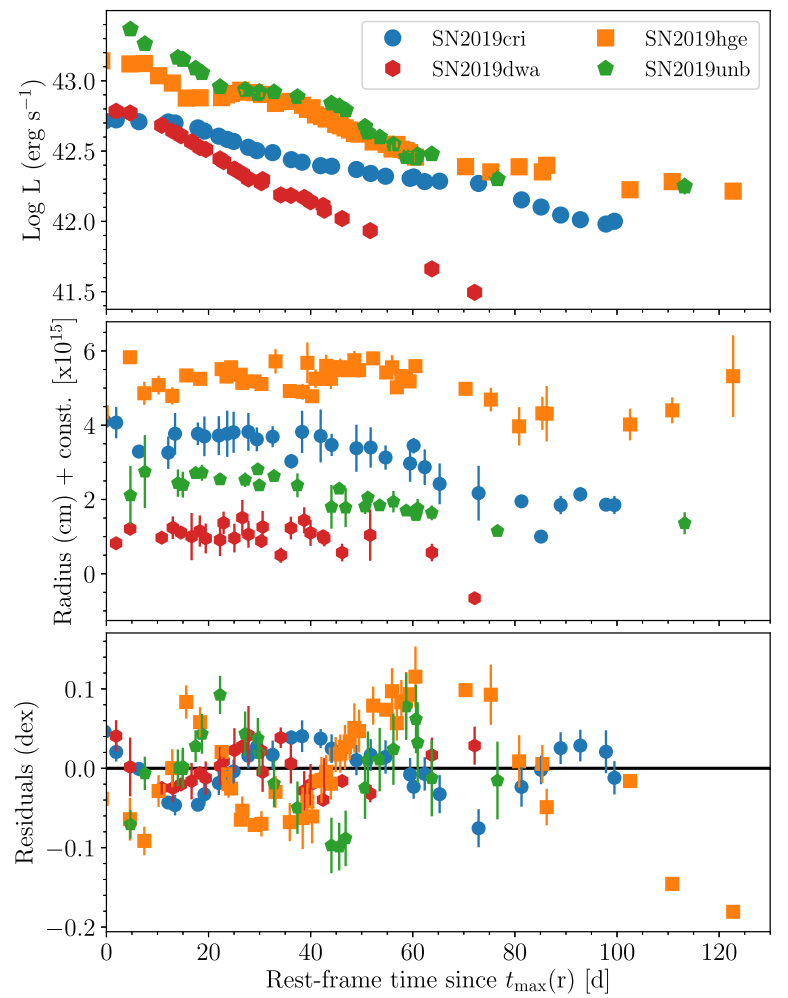

Figure 17. Upper panel: after peak bolometric light curves of our transitional objects. Middle panel: photospheric radius evolution as derived from blackbody fitting of the SED. Lower panel: residuals, after subtracting fits to the declining bolometric light curve.

Inserra et al. 2017). To explore this further, we fitted a first-order polynomial to the bolometric light curve from the peak to the end of the available photometry. The choice of using the bolometric allows us to avoid an analysis dominated by line evolution within a particular passband. The bolometric light curves (cfr. Fig. 17, top panel) were built following the procedure outlined in Inserra et al. (2018a). The choice of a linear fit is due to the fact that SE-SNe are usually well reproduced by models having a steady decline over the available time-scale. The residuals in Fig. 17 (bottom panel) show clear fluctuation of the order of roughly 0.1 dex for SN 2019hge (at $\sim 20$ and $\sim 70 \mathrm{~d}$ ) and SN 2019 unb (at $\sim 20$ and $\sim 60 \mathrm{~d}$ ) and possibly also in SN 2019cri at $\sim 70 \mathrm{~d}$. Nothing conclusive can be said for SN 2019dwa as any possible fluctuation is at the same level of the uncertainties. This reflects what observed in the single broad-band filters. Such fluctuations are a factor of 10 larger than the uncertainties, and roughly a factor of 2 larger than what observed in 'slow' SLSNe I (e.g. Nicholl et al. 2016a; Inserra et al. 2017; Inserra 2019).

In a normal SN scenario, such fluctuations might be caused by a change in the density profile of the emitting region. Such a change is also usually reflected by a change in the radius evolution of the photosphere (Liu et al. 2018). Hence, we derived the radius evolution via the Stefan-Boltzmann law and using as input the bolometric light curve and the temperature evolution derived by fitting the available spectra energy distribution with a Planck function. The radius evolution displayed in Fig. 17 (middle panel) do not show any stark change in the radius evolution. Nevertheless, a change in the line/ions is observed in the spectra evolution. Another viable alternative to produce such undulations is via interaction with a small amount of CSM material as observed in interacting transients (e.g. Smith et al. 2012). Using the scaling relation presented by

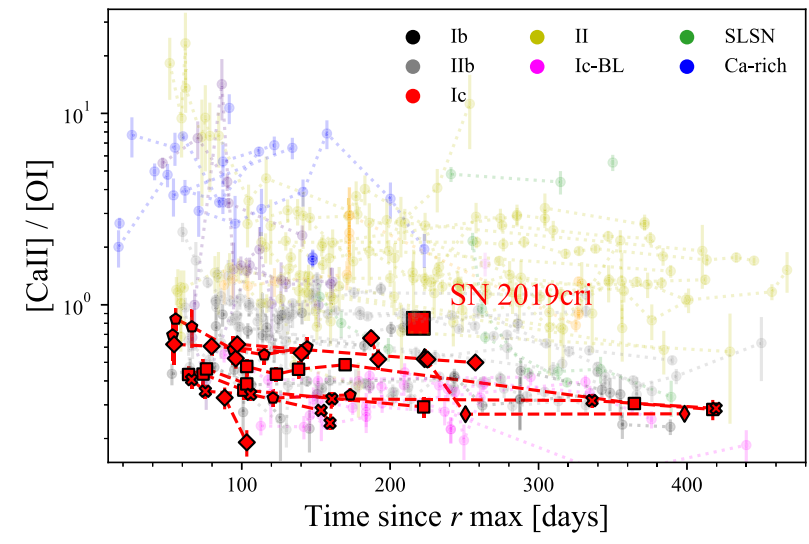

Figure 18. The [Ca II] $\lambda \lambda 7291,7323 /[\mathrm{O}$ I] $\lambda \lambda 6300,6364$ plane (Prentice et al., submitted) with SN2019cri (red square) in context against SNe Ic (red) and other transients (see legend). The ratio of SN 2019cri is marginally larger than SNe Ic at the same epoch. It may be expected however, that if this ratio is a measure of core oxygen mass, that SN 2019cri would be lower than the other transients based upon the light curve and snapshot spectral model, which would indicate a relatively large $\mathrm{O}$ mass.

Smith \& McCray (2007), $L \approx M_{\mathrm{CSM}} v^{2} / 2 t$ and assuming an average half-period of the fluctuation of $t=10 \mathrm{~d}$ (only the fluctuation at $70 \mathrm{~d}$ of SN 2019hge has half-period of $20 \mathrm{~d}$ ), average luminosity of $L=10^{43} \mathrm{erg} \mathrm{s}^{-1}$ and velocity of $5000 \mathrm{~km} \mathrm{~s}^{-1}$, we obtain CSM masses of $0.03-0.07 \mathrm{M}_{\odot}$. As this seems periodic, they might be the consequence of a close binary scenario for the progenitor, which cause a heterogeneous structure of the CSM and could also explain the absence of interaction signature in the spectra due to a clumpy structure or viewing angle (Moriya et al. 2015).

\subsection{SN 2019cri and Ic-7}

The narrow lines of SNe Ic-7 are indicative of steep ejecta density profiles which results in low specific kinetic energy. The model spectrum presented here of SN 2019cri agrees with the $E_{\mathrm{k}} / M_{\mathrm{ej}}$ found for SN2017ein by Teffs et al. (2021). It becomes difficult to explain why objects with such diverse photometric properties should display so similar spectroscopic properties. The possible powering mechanisms for the four $\mathrm{SNe}$ featured in this work were discussed previously, and it was stated that the light curve of SN 2019cri is incompatible with a pure ${ }^{56} \mathrm{Ni}$ decay model. The question then arises; if SN 2019cri need not be powered primarily by ${ }^{56} \mathrm{Ni}$ then why should the other objects of this class? It then becomes pertinent to examine SN $2011 \mathrm{bm}$ in this context. This transient requires $\sim 0.7 \mathrm{M}_{\odot}$ of ${ }^{56} \mathrm{Ni}$ to power its peak luminosity, comparable to $\mathrm{SNe} \mathrm{Ia}$, and $7-11 \mathrm{M}_{\odot}$ of ejecta (via Arnett Valenti et al. 2012) to display such a broad light curve. Prentice et al. (submitted) show that if the ratio of [Ca II] $\lambda \lambda 7292,7324$ to [O I] $\lambda \lambda 6300,6364$ for this object is compared with other SE-SNe, where the ejecta masses are typically $\sim 3 \mathrm{M}_{\odot}$, then SN 2011 bm takes the same ratio as low mass objects. In Section 4.2, a value of $\sim 0.8$ was calculated for SN 2019cri. Fig. 18 shows that this objects sits above is an outlier compared with other SNe Ic. This ratio is assumed to trace oxygen core mass (Fransson \& Chevalier 1987), which itself is a proxy for $M_{\mathrm{ej}}$ in stripped supernovae. Prentice et al. (submitted) suggest that one possible reason for this is that the powering mechanism of SN $2011 \mathrm{bm}$ is not predominantly via the ${ }^{56} \mathrm{Ni}$ decay chain and also pointed to lack of strong Fe II and Fe III emission lines in its nebular spectra. In SN 2019cri, we find an object that is more extreme, where ${ }^{56} \mathrm{Ni}$ is disfavoured as the 
primary powering source. Fortunately, this transient was followed into the early nebular phase. By taking the $[\mathrm{CaII}] /[\mathrm{OI}]$ ratio this object can be placed in context, and it is found that the ratio is even larger than for the SE-SNe. This could suggest that the slow evolution of this object is not due to a large ejecta mass, but perhaps a dense wall within the ejecta profile, perhaps blown by a magnetar (Kasen \& Bildsten 2010). Another possibility is that the [Ca II] line is contaminated or even dominated by [O II], artificially inflating the $[\mathrm{CaII}] /[\mathrm{OI}]$ ratio, if oxygen in the ejecta remains ionized into the nebular phase (Jerkstrand et al. 2017). This can occur due to 'freezein' of the ionization state in ejecta heated by a power-law central engine (Margalit et al. 2018).

\section{CONCLUSIONS}

SN 2019unb and SN2019hge have both been classified as SLSNe, yet, photometrically they are close to $\mathrm{SNe}$ Ibc in terms of luminosity (they sit close to the $M=-19.8$ mag boundary defined by Gal-Yam 2012), strengthening the case that a more robust definition, ideally linked to the spectroscopic evolution, is needed (Gal-Yam 2019; Inserra 2019). Spectroscopically, they share few similarities with the canonical SLSN, they lack the O II and C II lines seen in their early spectra, but they do not share the same evolution time-scales as they are more similar to $\mathrm{SNe} \mathrm{Ib} / \mathrm{IIb}$ at later times than normal SLSN. It was noted that convolving a blackbody with an $\mathrm{SN}$ Ib spectrum gave a good approximation to the early spectra of SN 2019unb and spectral modelling suggests a $10 \mathrm{M}_{\odot}$ ejecta and normal relative abundances compared with $\mathrm{SN}$ Ib.

SNe 2019cri and 2019dwa, are similar to SE-SNe, especially SN Ic-7; displaying narrow Fe II lines. Snapshot spectral modelling of the maximum light spectra suggests these transients may have large ejecta masses of 10 and $6 \mathrm{M}_{\odot}$, respectively, and a specific kinetic energy of $\sim 1$, although we add a note of caution with these results. It also shows that SN 2019cri evolves spectroscopically on a time-scale typical of SE-SNe after maximum and that the appearance of [OI] $\lambda \lambda 6300,6364$ coincides with a drop in luminosity. We suggest that this may be evidence for a density 'wall' within the ejecta, whereby the line-forming region drops from a high- to low-density region and so the spectra change appearance relatively rapidly. This is analogous to the bubble blown by a pulsar wind and has been shown to occur in the presence of a magnetar.

The variability and luminosity of the light curves preclude powering from a purely radioactive source, while the variability itself suggests either variation in the powering source or a non-monotonic ejecta density profile. Given the absence of any clear emission lines associated with CSM interaction in any of the objects, we suggest that the powering mechanism for the light curve and ejecta dynamics is likely dominated by energy from a compact source.

It is demonstrated that the hosts of these $\mathrm{SNe}$ are consistent with that of SLSNe; low mass and high star formation rates, with the exception of SN2019hge, which was hosted in a high-mass galaxy with a high star formation rate. Considering the inferred ejecta masses along with the presence of these events in star-forming galaxies, it is likely that the progenitors of these objects are more massive than those typically associated with SE-SNe and more akin to SLSNe.

If these objects are to be placed within the existing taxonomy, the classification should reflect physical parameters of the objects. In this case, we can summarize these two events as follows:

(i) They have He-rich ejecta, possibly also a thin $\mathrm{H}$ shell.

(ii) They have extremely low ejecta velocities in comparison with other SNe. (iii) Their light curves are luminous and variable which might be a hint of a binary system scenario (for at least some of them).

(iv) Their early spectra are hot; and their early colour evolution suggests this is the case for many weeks.

(v) Spectroscopically, they display no obvious signs of CSM interaction.

We can only hypothesize as to what powers these events; and there exists no theoretical model to explain their observed properties. Given the spectroscopic similarity with $\mathrm{SNe} \mathrm{Ib} / \mathrm{IIb}$, it may be useful to consider than as a peculiar sub-class of these events. It would also not be the first time that $\mathrm{SNe} \mathrm{Ib} / \mathrm{IIb}$ have displayed unusual photometric properties. Prior to this was the case of SN 2005bf and its double-peaked light curve that reached $M_{r} \sim-18.5$ in nearly $60 \mathrm{~d}$.

An increasing number of these rare objects are now being found and prove the diversity of SNe between SLSNe I and SE-SNe. To understand them, we need to address the following questions: do SE-SNe and SLSNe represent a continuous class of objects as suggested by the luminosity function, or are they separable, as suggested by their spectroscopic sequence? Can high-resolution spectroscopy show a relationship between the ejecta velocity and light-curve variation? If so, this could represent deviation away from a power-law ejecta density profile.

\section{ACKNOWLEDGEMENTS}

KM, MRM, and SJP are supported by H2020 ERC grant no. 758638. LG acknowledges financial support from the European Union's Horizon 2020 research and innovation programme under the Marie Skłodowska-Curie grant agreement no. 839090, and from the Spanish Ministry of Science, Innovation and Universities (MICIU) under the 2019 Ramón y Cajal programme RYC2019-027683. TMB was funded by the CONICYT PFCHA / DOCTORADOBECAS CHILE/2017-72180113. MG is supported by the EU Horizon 2020 research and innovation programme under grant agreement no. 101004719. SGG acknowledges support by FCT under Project CRISP PTDC/FIS-AST-31546/2017. MN is supported by a Royal Astronomical Society Research Fellowship and H2020 ERC grant no. 948381. T-WC acknowledges the EU Funding under Marie Skłodowska-Curie grant H2020-MSCA-IF-2018-842471. The LT is operated on the island of La Palma by Liverpool John Moores University in the Spanish Observatorio del Roque de los Muchachos of the Instituto de Astrofisica de Canarias with financial support from the UK Science and Technology Facilities Council. Based on observations collected at the European Organisation for Astronomical Research in the Southern Hemisphere, Chile, as part of ePESSTO+ (the advanced Public ESO Spectroscopic Survey for Transient Objects Survey). ePESSTO+ observations were obtained under ESO programme ID 1103.D-0328 (PI: Inserra). The WHT is operated on the island of La Palma by the Isaac Newton Group of Telescopes in the Spanish Observatorio del Roque de los Muchachos of the Instituto de Astrofísica de Canarias. SJP thanks GPL for many insightful discussions at the bar over the last few years.

\section{DATA AVAILABILITY}

Data will be made available on the WISeREP at www.wiserep.org.

\section{REFERENCES}

Ahn C. P. et al., 2012, ApJS, 203, 21

Ahn C. P. et al., 2014, ApJS, 211, 17 
Angus C. R. et al., 2019, MNRAS, 487, 2215

Arnett W. D., 1982, ApJ, 253, 785

Ashall C. et al., 2019, MNRAS, 487, 5824

Ashall C., Mazzali P. A., 2020, MNRAS, 492, 5956

Barnsley R. M., Smith R. J., Steele I. A., 2012, Astron. Nachr., 333, 101

Bellm E. C. et al., 2019, PASP, 131, 018002

Bose S. et al., 2018, ApJ, 853, 57

Bourne N. et al., 2012, MNRAS, 421, 3027

Breeveld A. A., Landsman W., Holland S. T., Roming P., Kuin N. P. M., Page M. J., 2011, in McEnery J. E., Racusin J. L., Gehrels N., eds, AIP Conf. Proc. Vol.1358, GAMMA RAY BURSTS. Am. Inst. Phys., New York, p. 373

Buzzoni B. et al., 1984, The Messenger, 38, 9

Byler N., Dalcanton J. J., Conroy C., Johnson B. D., 2017, ApJ, 840, 44

Calzetti D., Armus L., Bohlin R. C., Kinney A. L., Koornneef J., StorchiBergmann T., 2000, ApJ, 533, 682

Cardelli J. A., Clayton G. C., Mathis J. S., 1989, ApJ, 345, 245

Chabrier G., 2003, PASP, 115, 763

Chambers K. C. et al., 2016, preprint (arXiv:1612.05560)

Chen K.-J., Woosley S. E., Sukhbold T., 2016, ApJ, 832, 73

Chen T. W. et al., 2018, ApJ, 867, L31

Chen T.-W., Smartt S. J., Yates R. M., Nicholl M., Krühler T., Schady P., Dennefeld M., Inserra C., 2017, MNRAS, 470, 3566

Dahiwale A., Dugas A., Fremling C., 2019, TNS Class. Rep., 2019, 1

Dahiwale A., Fremling C., 2020, TNS Class. Rep., 2020, 1

De Cia A. et al., 2018, ApJ, 860, 100

Dey A. et al., 2019, AJ, 157, 168

Drout M. R. et al., 2011, ApJ, 741, 97

Filippenko A. V., 1997, ARA\&A, 35, 309

Fransson C., Chevalier R. A., 1987, ApJ, 322, L15

Fremling C., Dugas A., Sharma Y., 2019, TNS Class. Rep., 2019, 1

Frohmaier C. et al., 2021, MNRAS, 500, 5142

Gal-Yam A., 2012, Science, 337, 927

Gal-Yam A., 2019, ARA\&A, 57, 305

Gehrels N. et al., 2004, ApJ, 611, 1005

Gomez S., Berger E., Hosseinzadeh G., Blanchard P. K., Nicholl M., Villar V. A., 2021, ApJ, 913, 14

Hachinger S., Mazzali P. A., Taubenberger S., Pakmor R., Hillebrandt W., 2009, MNRAS, 399, 1238

Hinshaw G. et al., 2013, ApJS, 208, 19

Hosseinzadeh G. et al., 2017, ApJ, 836, 158

Hunter D. J. et al., 2009, A\&A, 508, 371

Inserra C. et al., 2013, ApJ, 770, 128

Inserra C. et al., 2017, MNRAS, 468, 4642

Inserra C. et al., 2018a, MNRAS, 475, 1046

Inserra C., 2019, Nature Astron., 3, 697

Inserra C., Prajs S., Gutierrez C. P., Angus C., Smith M., Sullivan M., 2018b, ApJ, 854, 175

Inserra C., Smartt S. J., 2014, ApJ, 796, 87

Jerkstrand A. et al., 2017, ApJ, 835, 13

Kasen D., Bildsten L., 2010, ApJ, 717, 245

Khatami D. K., Kasen D. N., 2019, ApJ, 878, 56

Lang D., 2014, AJ, 147, 108

Leja J., Johnson B. D., Conroy C., van Dokkum P. G., Byler N., 2017, ApJ, 837,170

Leloudas G. et al., 2015, MNRAS, 449, 917

Liu L.-D., Zhang B., Wang L.-J., Dai Z.-G., 2018, ApJ, 868, L24

Liu Y.-Q., Modjaz M., Bianco F. B., 2017a, ApJ, 845, 85

Liu Y.-Q., Modjaz M., Bianco F. B., 2017b, ApJ, 845, 85

Lucy L. B., 1999, A\&A, 345, 211

Lunnan R. et al., 2020, ApJ, 901, 61

Lyman J. D., Bersier D., James P. A., Mazzali P. A., Eldridge J. J., Fraser M., Pian E., 2016, MNRAS, 457, 328

Mainzer A. et al., 2014, ApJ, 792, 30

Margalit B., Metzger B. D., Berger E., Nicholl M., Eftekhari T., Margutti R., 2018, MNRAS, 481, 2407

Martin D. C. et al., 2005, ApJ, 619, L1

Matheson T. et al., 2000, AJ, 120, 1487
Mazzali P. A. et al., 2002, ApJ, 572, L61

Mazzali P. A., 2000, A\&A, 363, 705

Mazzali P. A., Lucy L. B., 1993, A\&A, 279, 447

Mazzali P. A., Sauer D. N., Pian E., Deng J., Prentice S., Ben Ami S., Taubenberger S., Nomoto K., 2017, MNRAS, 469, 2498

Mazzali P. A., Sullivan M., Pian E., Greiner J., Kann D. A., 2016, MNRAS, 458,3455

Mazzali P. A., Walker E. S., Pian E., Tanaka M., Corsi A., Hattori T., Gal-Yam A., 2013, MNRAS, 432, 2463

Meisner A. M., Lang D., Schlegel D. J., 2017, AJ, 153, 38

Modjaz M., Gutiérrez C. P., Arcavi I., 2019, Nature Astron., 3, 717

Moriya T. J., Liu Z.-W., Mackey J., Chen T.-W., Langer N., 2015, A\&A, 584, L5

Müller B., 2015, MNRAS, 453, 287

Nicholl M. et al., 2015, MNRAS, 452, 3869

Nicholl M. et al., 2016a, ApJ, 826, 39

Nicholl M. et al., 2016b, ApJ, 828, L18

Nicholl M., Berger E., Blanchard P. K., Gomez S., Chornock R., 2019, ApJ, 871,102

Nomoto K., Yamaoka H., Pols O. R., van den Heuvel E. P. J., Iwamoto K., Kumagai S., Shigeyama T., 1994, Nature, 371, 227

Nyholm A. et al., 2020, A\&A, 637, A73

Parrag E. et al., 2021, MNRAS, 506, 4819

Pastorello A. et al., 2010, ApJ, 724, L16

Pauldrach A. W. A., Duschinger M., Mazzali P. A., Puls J., Lennon M., Miller D. L., 1996, A\&A, 312, 525

Perley D. A. et al., 2016, ApJ, 830, 13

Pian E. et al., 2020, MNRAS, 497, 3542

Piascik A. S., Steele I. A., Bates S. D., Mottram C. J., Smith R. J., Barnsley R. M., Bolton B., 2014, in Ramsay S. K., McLean I. S., Takami H., eds, SPIE Conf. Ser. Vol. 9147, Ground-Based and Airborne Instrumentation for Astronomy V. SPIE, Belligham, p. $91478 \mathrm{H}$

Poznanski D., Prochaska J. X., Bloom J. S., 2012, MNRAS, 426, 1465

Prentice S. J. et al., 2016, MNRAS, 458, 2973

Prentice S. J. et al., 2019b, MNRAS, 485, 1559

Prentice S. J., Maguire K., Skillen K., Magee M. R., Clark P., 2019a, TNS AstroNote, 74, 1

Prentice S. J., Maguire K., Skillen K., Magee M. R., Clark P., 2019c, TNS Disc. Rep., 2019, 1

Prentice S. J., Maguire K., Skillen K., Magee M. R., Clark P., 2019d, TNS Class. Rep., 2019, 1

Prentice S. J., Maguire K., Skillen K., Magee M. R., Clark P., 2019e, TNS Class. Rep., 2019, 1

Prentice S. J., Mazzali P. A., 2017, MNRAS, 469, 2672

Quimby R. M. et al., 2011, Nature, 474, 487

Quimby R. M. et al., 2018, ApJ, 855, 2

Quimby R. M., Yuan F., Akerlof C., Wheeler J. C., 2013, MNRAS, 431, 912

Roming P. W. A. et al., 2005, Space Sci. Rev., 120, 95

Roy R. et al., 2016, A\&A, 596, A67

Sauer D. N., Mazzali P. A., Deng J., Valenti S., Nomoto K., Filippenko A. V., 2006, MNRAS, 369, 1939

Schlafly E. F., Finkbeiner D. P., 2011, ApJ, 737, 103

Schulze S. et al., 2021 ,ApJ, 255,29

Schulze S. et al., 2018, MNRAS, 473, 1258

Schulze S. et al., 2020a, ApJ, 255, 35

Shivvers I. et al., 2019, MNRAS, 482, 1545

Skrutskie M. F. et al., 2006, AJ, 131, 1163

Smartt S. J. et al., 2015, A\&A, 579, A40

Smith N., Mauerhan J. C., Silverman J. M., Ganeshalingam M., Filippenko A. V., Cenko S. B., Clubb K. I., Kandrashoff M. T., 2012, MNRAS, 426, 1905

Smith N., McCray R., 2007, ApJ, 671, L17

Steele I. A. et al., 2004, in Jacobus M., Oschmann J., ed., Proc. SPIE Conf. Ser. Vol. 5489, Ground-Based Telescopes. SPIE, Bellingham, 679

Stehle M., Mazzali P. A., Benetti S., Hillebrandt W., 2005, MNRAS, 360, 1231

Stritzinger M. D. et al., 2018, A\&A, 609, A135

Stritzinger M., Leibundgut B., 2005, A\&A, 431, 423 
Suzuki A., Maeda K., 2019, ApJ, 880, 150

Suzuki A., Maeda K., 2021, ApJ, 908, 217

Taddia F. et al., 2018, A\&A, 609, A136

Taubenberger S. et al., 2009, MNRAS, 397, 677

Teffs J. J., Prentice S. J., Mazzali P. A., Ashall C., 2021, MNRAS, 502, 3829

Valenti S. et al., 2011, MNRAS, 416, 3138

Valenti S. et al., 2012, ApJ, 749, L28

Wiseman P. et al., 2020, MNRAS, 495, 4040

Wright A. H. et al., 2016, MNRAS, 460, 765

Wright E. L. et al., 2010, AJ, 140, 1868

Yan L. et al., 2020, ApJ, 902, L8

\section{APPENDIX A: SN 2019YZ}

SN 2019yz is a spectroscopically and photometrically normal Type Ic-7, and is provided here as a comparison object. We present a quick summary of the properties of this transient in Table A1. The distance
Table A1. The properties of SN 2019yz.

\begin{tabular}{lcccc}
\hline $\mathrm{SN}$ & $z$ & $\begin{array}{c}\mu \\
(\mathrm{mag})\end{array}$ & $\begin{array}{c}E(B-V)_{\mathrm{MW}} \\
(\mathrm{mag})\end{array}$ & $\begin{array}{c}E(B-V)_{\text {host }} \\
(\mathrm{mag})\end{array}$ \\
\hline $2019 \mathrm{yz}$ & 0.006 & 32.3 & 0.1 & $0.2 \pm 0.1$ \\
\hline
\end{tabular}

modulus is a Tully-Fisher measurement, while $E(B-V)_{\mathrm{Mw}}$ is from Schlafly \& Finkbeiner (2011). The host extinction is estimated by fitting two Gaussian profiles to the host Na I D absorption lines and applying the relationship between the pseudo-equivalent width and reddening from Poznanski, Prochaska \& Bloom (2012). The data for this object will be made public along with the SNe featured in this work.

This paper has been typeset from a $\mathrm{T}_{\mathrm{E}} \mathrm{X} / \mathrm{L} \mathrm{T} \mathrm{E} \mathrm{X}$ file prepared by the author. 